\title{
Modeling the distribution of ranks, Selmer groups, and Shafarevich-Tate groups of elliptic curves
}

\author{
Manjul Bhargava*, Daniel M. Kane ${ }^{\dagger}$, Hendrik W. Lenstra Jr., \\ BJORn POONEN ${ }^{\ddagger}$, AND ERIC RAINS
}

Using maximal isotropic submodules in a quadratic module over $\mathbb{Z}_{p}$, we prove the existence of a natural discrete probability distribution on the set of isomorphism classes of short exact sequences of cofinite type $\mathbb{Z}_{p}$-modules, and then conjecture that as $E$ varies over elliptic curves over a fixed global field $k$, the distribution of

$$
0 \rightarrow E(k) \otimes \mathbb{Q}_{p} / \mathbb{Z}_{p} \rightarrow \operatorname{Sel}_{p} E \rightarrow \amalg\left[p^{\infty}\right] \rightarrow 0
$$

is that one. We show that this single conjecture would explain many of the known theorems and conjectures on ranks, Selmer groups, and Shafarevich-Tate groups of elliptic curves. We also prove the existence of a discrete probability distribution on the set of isomorphism classes of finite abelian $p$-groups equipped with a nondegenerate alternating pairing, defined in terms of the cokernel of a random alternating matrix over $\mathbb{Z}_{p}$, and we prove that the two probability distributions are compatible with each other and with Delaunay's predicted distribution for Ш. Finally, we prove new theorems on the fppf cohomology of elliptic curves in order to give further evidence for our conjecture.

\section{Introduction}

\subsection{Selmer and Shafarevich-Tate groups}

Fix a global field $k$. Let $\Omega$ be the set of nontrivial places of $k$. Let $\mathscr{E}$ be the set of elliptic curves over $k$, or more precisely, a set containing one representative of each isomorphism class. Given $E \in \mathscr{E}$ and a positive integer $n$, the $n$-Selmer

\footnotetext{
* Supported by a Simons Investigator Award and the National Science Foundation grant DMS-1001828.

${ }^{\dagger}$ Supported by a National Science Foundation Graduate Fellowship.

¥Supported by the Guggenheim Foundation and National Science Foundation grants DMS-0841321 and DMS-1069236.
} 
group $\operatorname{Sel}_{n} E$ is a finite group that is used to bound the rank of the finitely generated abelian group $E(k)$. If $n$ is a product of prime powers $p^{e}$, then $\operatorname{Sel}_{n} E$ is the direct sum of the $\operatorname{Sel}_{p^{e}}$, so we focus on the latter groups. If $p$ is prime, one may also form the direct limit $\operatorname{Sel}_{p^{\infty}} E:=\lim \operatorname{Sel}_{p^{e}} E$. This group, together with the $p$-primary subgroup of the Shafarevich-Tate group $\amalg=$ $\amalg(E):=\operatorname{ker}\left(\mathrm{H}^{1}(k, E) \rightarrow \prod_{v \in \Omega} \mathrm{H}^{1}\left(k_{v}, E\right)\right)$, fits into an exact sequence

$\left(\mathrm{Seq}_{E}\right) \quad 0 \longrightarrow E(k) \otimes \frac{\mathbb{Q}_{p}}{\mathbb{Z}_{p}} \longrightarrow \operatorname{Sel}_{p^{\infty}} E \longrightarrow \amalg\left[p^{\infty}\right] \longrightarrow 0$

of $\mathbb{Z}_{p}$-modules.

Question 1.1. Given a short exact sequence $\mathscr{S}$ of $\mathbb{Z}_{p^{-}}$-modules, what is the probability that $\mathrm{Seq}_{E} \simeq \mathscr{S}$ as $E$ varies over $\mathscr{E}$, ordered by height?

Our goal is to formulate a conjectural answer and to prove that it would imply many of the known theorems and conjectures on ranks, Selmer groups, and Shafarevich-Tate groups of elliptic curves. For example, it would imply that asymptotically $50 \%$ of elliptic curves over $k$ have rank $0,50 \%$ have rank 1, and $0 \%$ have rank 2 or more: see Section 5.3.

\subsection{Intersection of random maximal isotropic $\mathbb{Z}_{p}$-modules}

Let $n \in \mathbb{Z}_{\geq 0}$. Equip $V:=\mathbb{Z}_{p}^{2 n}$ with the standard hyperbolic quadratic form $Q: V \rightarrow \mathbb{Z}_{p}$ given by

$$
Q\left(x_{1}, \ldots, x_{n}, y_{1}, \ldots, y_{n}\right):=\sum_{i=1}^{n} x_{i} y_{i} .
$$

A $\mathbb{Z}_{p}$-submodule $Z$ of $V$ is called isotropic if $\left.Q\right|_{Z}=0$. Let $\mathrm{OGr}_{V}\left(\mathbb{Z}_{p}\right)$ be the set of maximal isotropic direct summands $Z$ of $V$; each such $Z$ is free of rank $n$. There is a natural probability measure on $\operatorname{OGr}_{V}\left(\mathbb{Z}_{p}\right)$, defined so that for each $e \geq 0$, the distribution of $Z / p^{e} Z$ in $V / p^{e} V$ is uniform among all possibilities (see Sections 2 and 4). Choose $Z, W \in \operatorname{OGr}_{V}\left(\mathbb{Z}_{p}\right)$ independently at random. View $Z \otimes \frac{\mathbb{Q}_{p}}{\mathbb{Z}_{p}}$ and $W \otimes \frac{\mathbb{Q}_{p}}{\mathbb{Z}_{p}}$ as $\mathbb{Z}_{p}$-submodules of $V \otimes \frac{\mathbb{Q}_{p}}{\mathbb{Z}_{p}}$, where the tensor products are over $\mathbb{Z}_{p}$. Define

$$
R:=(Z \cap W) \otimes \frac{\mathbb{Q}_{p}}{\mathbb{Z}_{p}} \quad \text { and } \quad S:=\left(Z \otimes \frac{\mathbb{Q}_{p}}{\mathbb{Z}_{p}}\right) \cap\left(W \otimes \frac{\mathbb{Q}_{p}}{\mathbb{Z}_{p}}\right)
$$

and define $T$ to complete an exact sequence

$$
0 \rightarrow R \rightarrow S \rightarrow T \rightarrow 0
$$


Then $R$ is a finite power of $\mathbb{Q}_{p} / \mathbb{Z}_{p}$, the module $T$ is finite, and the sequence splits: see Section 5.1. In particular, each of the $\mathbb{Z}_{p}$-modules $R, S, T$ is of co-finite type. (A $\mathbb{Z}_{p}$-module $M$ is of co-finite type if its Pontryagin dual is finitely generated over $\mathbb{Z}_{p}$, or equivalently if $M$ is isomorphic to $\left(\mathbb{Q}_{p} / \mathbb{Z}_{p}\right)^{s} \oplus F$ for some $s \in \mathbb{Z}_{\geq 0}$ and finite abelian $p$-group $F$.)

\section{Theorem 1.2.}

(a) As $Z$ and $W$ vary, the sequence $0 \rightarrow R \rightarrow S \rightarrow T \rightarrow 0$ defines a discrete probability distribution $\mathscr{Q}_{2 n}$ on the set of isomorphism classes of short exact sequences of co-finite type $\mathbb{Z}_{p}$-modules.

(b) The distributions $\mathscr{Q}_{2 n}$ converge to a discrete probability distribution $\mathscr{Q}$ as $n \rightarrow \infty$.

(Here $\mathscr{Q}$ is for "quadratic".) Theorem 1.2 will be proved in Section 5.4.

\subsection{The model}

Let $E \in \mathscr{E}$, and let $r$ be the rank of $E(k)$. Each term in $\mathrm{Seq}_{E}$ is a co-finite type $\mathbb{Z}_{p}$-module. In fact, $E(k) \otimes \frac{\mathbb{Q}_{p}}{\mathbb{Z}_{p}} \simeq\left(\frac{\mathbb{Q}_{p}}{\mathbb{Z}_{p}}\right)^{r}$ and $\amalg\left[p^{\infty}\right]$ is conjecturally finite. Moreover, since $E(k) \otimes \frac{\mathbb{Q}_{p}}{\mathbb{Z}_{p}}$ is divisible, the sequence (Seq $E$ ) splits.

Conjecture 1.3. Fix a global field $k$. For each short exact sequence $\mathscr{S}$ of $\mathbb{Z}_{p}$-modules, the density of $\left\{E \in \mathscr{E}: \mathrm{Seq}_{E} \simeq \mathscr{S}\right\}$ equals the $\mathscr{Q}$-probability of $\mathscr{S}$.

In other words, the sequence $0 \rightarrow R \rightarrow S \rightarrow T \rightarrow 0$ models $\mathrm{Seq}_{E}$. In particular, $R$ conjecturally measures the rank of the group of rational points, $S$ models the $p^{\infty}$-Selmer group, and $T$ models the $p$-primary part of the Tate-Shafarevich group.

Remark 1.4. To define density of a subset of $\mathscr{E}$ precisely, one orders $\mathscr{E}$ by height as explained in the introductions to [BS15a] and [PR12]. The reason for ordering $\mathscr{E}$ by height is that most other orderings lead to statements that are difficult to corroborate: for instance, the asymptotic behavior of the number of elliptic curves over $\mathbb{Q}$ of conductor up to $X$ is unknown, even when no condition on its Selmer sequence is imposed.

Remark 1.5. Certain restricted families of elliptic curves can exhibit very different Selmer group behavior. The average size of $\mathrm{Sel}_{2} E$ can even be infinite in certain families. See [Yu05], [XZ09], [XZ08], [FX12], and [KLO15] for work in this direction.

We will prove that Conjecture 1.3 has the following consequences, the first of which was mentioned already: 
- Asymptotically, $50 \%$ of elliptic curves over $k$ have rank 0 , and $50 \%$ have rank 1; cf. [Gol79, Conjecture B] and [KS99a, KS99b].

- $\amalg\left[p^{\infty}\right]$ is finite for $100 \%$ of elliptic curves over $k$.

- Conjecture 1.1(a) of [PR12] concerning the distribution of $\operatorname{Sel}_{p} E$ holds. In fact, our Conjecture 1.3 implies a generalization concerning the distribution of $\operatorname{Sel}_{p^{e}} E$ for every $e \geq 0$ (see Section 5.5).

- Delaunay's conjecture in [Del01, Del07, DJ14a] à la Cohen-Lenstra regarding the distribution of $\amalg\left[p^{\infty}\right]$ for rank $r$ elliptic curves over $\mathbb{Q}$ holds for $r=0$ and $r=1 .^{1}$

If we assume in addition that \# $\operatorname{Sel}_{p^{e}} E$ has bounded second moment, then it follows also that the average size of $\operatorname{Sel}_{p^{e}} E$ is $\sigma\left(p^{e}\right)$, the sum of the divisors of $p^{e}$; in fact, a generalization of our model, developed in Section 5.7, suggests that for every $n \geq 1$, the average size of $\operatorname{Sel}_{n} E$ is $\sigma(n)$. The same prediction was made in [BS13a, Conjecture 4], for different reasons, explained near the end of the introduction of [BS13a].

All these consequences are consistent with the partial results that have been proven, such as those in [BS15a, BS15b, BS13a, BS13b]. See also the introduction of [PR12] for discussion of related results.

For $r \geq 2$, Conjecture 1.3 cannot say anything about the distribution of $\amalg\left[p^{\infty}\right]$ as $E$ varies over the set $\mathscr{E}_{r}:=\{E \in \mathscr{E}: \operatorname{rk} E(k)=r\}$, because the locus of $(Z, W) \in \operatorname{OGr}_{V}\left(\mathbb{Z}_{p}\right)^{2}$ where $\operatorname{rk}(Z \cap W)=r$ is of measure 0 (Proposition 5.6). On the other hand, that locus carries another natural probability measure, so we may formulate a variant of Theorem 1.2:

\section{Theorem 1.6.}

(a) If we choose $(Z, W)$ at random from the locus in $\mathrm{OGr}_{V}\left(\mathbb{Z}_{p}\right)^{2}$ where $\operatorname{rk}(Z \cap W)=r$, then the isomorphism type of $T$ is given by a discrete probability distribution $\mathscr{T}_{2 n, r}$.

(b) The distributions $\mathscr{T}_{2 n, r}$ converge to a limit $\mathscr{T}_{r}$ as $n \rightarrow \infty$.

(c) The distribution $\mathscr{T}_{r}$ is the same as the distribution in Delaunay's conjecture on $\amalg\left[p^{\infty}\right]$ for rank $r$ elliptic curves over $\mathbb{Q}$.

Theorem 1.6 will be proved in Section 5.4.

\footnotetext{
${ }^{1}$ For this conjecture, see [Del01, Heuristic Assumption], with the modification that $u / 2$ is replaced by $u$, as suggested by the $u=1$ case discussed in [Del07, §3.2] (his $u$ is our $r$ ); see also [PR12, Section 6] and [DJ14a, Section 6.2]. Strictly speaking, in order to have our model match Delaunay's conjecture, we modify his conjecture to order elliptic curves over $\mathbb{Q}$ by height instead of conductor.
} 
Conjecture 1.7. Fix a global field $k$ and $r \in \mathbb{Z}_{\geq 0}$ such that $\mathscr{E}_{r}$ is infinite. For each finite abelian $p$-group $G$, the density of $\left\{E \in \mathscr{E}_{r}: \amalg\left[p^{\infty}\right] \simeq G\right\}$ in $\mathscr{E}_{r}$ equals the $\mathscr{T}_{r}$-probability of $G$.

Remark 1.8. Delaunay made predictions for the whole group Ш and not only $\amalg\left[p^{\infty}\right]$ for one $p$ at a time. We too will formulate a model for the whole of $\amalg$, naturally extending $\mathscr{T}_{r}$, and we will prove its compatibility with Delaunay's predictions: see Section 5.6.

Remark 1.9. Delaunay's conjecture for $\amalg\left[p^{\infty}\right]$ together with the conjecture that the rank $r$ is 0 or 1 with $50 \%$ probability each predicts conjectural distributions for $\operatorname{Sel}_{p^{\infty}} E$ and $\operatorname{Sel}_{p^{e}} E$ (cf. [DJ14a, §6.2] and [DJ14b, §5]). Specifically, $\operatorname{Sel}_{p^{\infty}} E \simeq\left(\mathbb{Q}_{p} / \mathbb{Z}_{p}\right)^{r} \oplus \amalg\left[p^{\infty}\right]$; if moreover $E(k)_{\text {tors }}=0$, as holds for $100 \%$ of elliptic curves (Lemma 5.7), then $\operatorname{Sel}_{p_{\infty}} E$ determines $\operatorname{Sel}_{p^{e}} E$ (Proposition 5.9(c)). This conjectural distribution for $\operatorname{Sel}_{p} E$ agrees with the $\mathscr{Q}$-distribution of $S$.

\subsection{Cokernel of a random alternating matrix}

Moreover, Conjecture 1.7 implies that another natural distribution on finite abelian $p$-groups yields a model for $\amalg\left[p^{\infty}\right]$. We now describe it. For an even integer $n$, choose an alternating $n \times n$ matrix $A \in M_{n}\left(\mathbb{Z}_{p}\right)$ (see Section 3.1 for definitions) at random with respect to Haar measure, and let $\mathscr{A}_{n, 0}$ be the distribution of coker $A$, which will turn out to be finite with probability 1 .

More generally, for fixed $r, n \geq 0$ with $n-r \in 2 \mathbb{Z}_{\geq 0}$, choose $A$ at random from the set of alternating matrices in $M_{n}\left(\mathbb{Z}_{p}\right)$ such that $\mathrm{rk} A=n-r$ (with respect to a measure to be described), and let $\mathscr{A}_{n, r}$ be the distribution of $(\operatorname{coker} A)_{\text {tors }}$.

Theorem 1.10. For each $r \geq 0$,

(a) the distributions $\mathscr{A}_{n, r}$ converge to a limit $\mathscr{A}_{r}$ as $n \rightarrow \infty$ through integers with $n-r \in 2 \mathbb{Z}_{>0}$, and

(b) the distributions $\mathscr{A}_{r}$ and $\mathscr{T}_{r}$ coincide.

Remark 1.11. Delaunay's conjecture for $\amalg$ was made in analogy with the Cohen-Lenstra heuristics for class groups [CL84]. Later, Friedman and Washington [FW89] recognized the Cohen-Lenstra distribution on p-primary parts as being the distribution of the cokernel of a random matrix over $\mathbb{Z}_{p}$. This was our motivation for modeling $\amalg\left[p^{\infty}\right]$ as the cokernel of a random alternating matrix over $\mathbb{Z}_{p}$. 


\subsection{Cassels-Tate pairing}

The Cassels-Tate pairing on $\amalg$ is an alternating bilinear pairing

$$
\amalg \times \amalg \rightarrow \mathbb{Q} / \mathbb{Z}
$$

whose kernel on each side is the maximal divisible subgroup of Ш. Taking $p$-primary parts yields an alternating bilinear pairing

$$
\amalg\left[p^{\infty}\right] \times \amalg\left[p^{\infty}\right] \rightarrow \mathbb{Q}_{p} / \mathbb{Z}_{p} .
$$

If $\amalg\left[p^{\infty}\right]$ is finite, then this pairing is nondegenerate.

Recall that we are modeling $\amalg\left[p^{\infty}\right]$ by the random groups $T$ and ( coker $A)_{\text {tors }}$ constructed in Sections 1.2 and 1.4, respectively. As evidence that these models are reasonable, we will construct a canonical nondegenerate alternating pairing on each of $T$ and ( $\operatorname{coker} A)_{\text {tors }}$; see Section 5.2.

\subsection{Arithmetic justification}

We have used theorems on the arithmetic of elliptic curves to guide the development of our models for ranks, Selmer groups, and Shafarevich-Tate groups. Conversely, part of the reason for developing such models is to suggest new structure in the arithmetic of elliptic curves that might be discovered.

In [PR12], modeling $\operatorname{Sel}_{p} E$ by an intersection of two random maximal isotropic $\mathbb{F}_{p}$-subspaces was suggested by a theorem that $\operatorname{Sel}_{p} E$ is an intersection of two maximal isotropic subspaces in an infinite-dimensional quadratic space over $\mathbb{F}_{p}$. In Section 6, we prove an analogue for prime powers: for $100 \%$ of $E \in \mathscr{E}$, the group $\operatorname{Sel}_{p^{e}} E$ is isomorphic to an intersection of two maximal isotropic subgroups of an infinite quadratic $\mathbb{Z} / p^{e} \mathbb{Z}$-module $\mathrm{H}^{1}\left(\mathbf{A}, E\left[p^{e}\right]\right)$. But why in our model do we assume that the two subgroups we intersect are direct summands? Answer: if maximal isotropic subgroups of $\left(\mathbb{Z} / p^{e} \mathbb{Z}\right)^{2 n}$ are to be sampled from a distribution that is invariant under the orthogonal group, then the only distribution that yields predictions compatible with the distribution in [PR12] modeling $\operatorname{Sel}_{p}$ is the distribution supported on direct summands (Remark 6.12). Moreover, we provide some justification from the arithmetic of elliptic curves: we prove that one of the two subgroups of $\mathrm{H}^{1}\left(\mathbf{A}, E\left[p^{e}\right]\right.$ ) actually is a direct summand (Corollary 6.8), and conjecture that the other one is too for $100 \%$ of $E \in \mathscr{E}$ (Conjecture 6.9). All this is the motivation for our model for $\mathrm{Sel}_{p^{\infty}}$.

Venkatesh and Ellenberg [VE10, Section 4.1] observed that the FriedmanWashington reinterpretation of the Cohen-Lenstra distribution could be jus- 
tified by a parallel construction in the arithmetic of number fields, namely that the class group is the cokernel of the homomorphism from the $S$-units to the group of fractional ideals supported on $S$, for a suitably large set of places $S$.

Question 1.12. Is there a construction in the arithmetic of elliptic curves that realizes $\amalg$ as the torsion subgroup of the cokernel of a natural alternating map of free $\mathbb{Z}$-modules?

\section{The canonical measure on the set of $\mathbb{Z}_{p}$-points of a scheme}

We will often need to choose points "uniformly at random" from the set of $\mathbb{Z}_{p}$-points of a scheme, e.g., the moduli space of $n \times n$ alternating matrices of rank $n-r$. The following proposition, a consequence of work of Oesterlé and Serre, explains what this means.

Proposition 2.1. Let $X$ be a finite-type $\mathbb{Z}_{p}$-scheme. Let $d=\operatorname{dim} X_{\mathbb{Q}_{p}}$. Equip $X\left(\mathbb{Z}_{p}\right)$ with the $p$-adic topology.

(a) There exists a unique bounded $\mathbb{R}_{\geq 0}$-valued measure $\mu=\mu_{X}$ on the Borel $\sigma$-algebra of $X\left(\mathbb{Z}_{p}\right)$ such that for any open and closed subset $S$ of $X\left(\mathbb{Z}_{p}\right)$, we have

$$
\mu(S)=\lim _{e \rightarrow \infty} \frac{\#\left(\text { image of } S \text { in } X\left(\mathbb{Z} / p^{e} \mathbb{Z}\right)\right)}{\left(p^{e}\right)^{d}} .
$$

(b) If $Y$ is a subscheme of $X$ and $\operatorname{dim} Y_{\mathbb{Q}_{p}}<d$, then $\mu\left(Y\left(\mathbb{Z}_{p}\right)\right)=0$.

(c) If $S$ is an open subset of $X\left(\mathbb{Z}_{p}\right)$, and $X_{\mathbb{Q}_{p}}$ is smooth of dimension $d$ at $s_{\mathbb{Q}_{p}}$ for some $s \in S$, then $\mu(S)>0$.

Proof.

(a) If $X$ is affine, this is a consequence of the discussion surrounding Théorème 2 of [Oes82], which builds on [Ser81, S3], and the Hahn-Kolmogorov extension theorem. In general, let $\left(X_{i}\right)$ be a finite affine open cover of $X$. Each set $X_{i}\left(\mathbb{Z}_{p}\right)$ is open and closed in $X\left(\mathbb{Z}_{p}\right)$, because $X_{i}\left(\mathbb{Z}_{p}\right)$ equals the inverse image of $X_{i}\left(\mathbb{F}_{p}\right)$ under the reduction map $X\left(\mathbb{Z}_{p}\right) \rightarrow X\left(\mathbb{F}_{p}\right)$. Since $\mathbb{Z}_{p}$ is a local ring, the sets $X_{i}\left(\mathbb{Z}_{p}\right)$ form a cover of $X\left(\mathbb{Z}_{p}\right)$. The measures on $X_{i}\left(\mathbb{Z}_{p}\right)$ and $X_{j}\left(\mathbb{Z}_{p}\right)$ are compatible on the intersection, by uniqueness, so they glue to give the required measure on $X\left(\mathbb{Z}_{p}\right)$.

(b) We may assume that $X$ is affine and that $Y$ is a closed subscheme of $X$. Even though $Y\left(\mathbb{Z}_{p}\right)$ might not be open in $X\left(\mathbb{Z}_{p}\right)$, it is an analytic closed subset (see [Oes82, §2]), so

$$
\mu\left(Y\left(\mathbb{Z}_{p}\right)\right)=\lim _{e \rightarrow \infty} \frac{\# Y\left(\mathbb{Z} / p^{e} \mathbb{Z}\right)}{\left(p^{e}\right)^{d}}
$$


still holds. According to [Ser81, p. 145, Théorème 8], \#Y(Z/Z/p $\mathbb{Z})=$ $O\left(\left(p^{e}\right)^{d-1}\right)$ as $e \rightarrow \infty$, so the limit is 0.

(c) See the discussion before Théorème 2 of [Oes82].

Corollary 2.2. If $X$ and $\mu$ are as in Proposition 2.1, and $X_{\mathbb{Q}_{p}}$ is smooth of dimension d at $x_{\mathbb{Q}_{p}}$ for some $x \in X\left(\mathbb{Z}_{p}\right)$, then $\mu$ can be normalized to yield a probability measure $\nu$ on $X\left(\mathbb{Z}_{p}\right)$.

From now on, when we speak of choosing an element of $X\left(\mathbb{Z}_{p}\right)$ uniformly at random for $X$ as in Corollary 2.2, we mean choosing it according to the measure $\nu$.

\section{Modeling Shafarevich-Tate groups using alternating matrices}

\subsection{Notation}

In this section, we assume that $R$ is a principal ideal domain. (Eventually we will take $R=\mathbb{Z}_{p}$. We could work with more general rings, but we have no need to.) Let $K=\operatorname{Frac} R$. Given an $R$-module $L$, let $L^{T}:=\operatorname{Hom}_{R}(L, R)$, let $L_{K}:=L \otimes_{R} K$, and let

$$
L_{\text {tors }}:=\{x \in L: r x=0 \text { for some nonzero } r \in R\}=\operatorname{ker}\left(L \rightarrow L_{K}\right) .
$$

Let $x . y$ denote the image of $(x, y)$ under the canonical pairing $L^{T} \times L \rightarrow R$, or $\left(L^{T}\right)_{K} \times L_{K} \rightarrow K$. If a free $R$-module $L$ has been fixed, and $N$ is a submodule of $L$, define the saturation

$$
N^{\text {sat }}:=N_{K} \cap L=\{x \in L: r x \in N \text { for some nonzero } r \in R\} .
$$

Given a homomorphism $A: L \rightarrow M$, let $A^{t}: M^{T} \rightarrow L^{T}$ denote the dual homomorphism; this notation is compatible with the notation $A^{t}$ for the transpose of a matrix. Let $M_{n}(R)_{\text {alt }}$ be the set of alternating $n \times n$ matrices, i.e., matrices $A$ with zeros on the diagonal satisfying $A^{t}=-A$. (The condition $A^{t}=-A$ already implies that the diagonal entries are 0 , except in characteristic 2.) For $S \subseteq M_{n}(R)$, define $S_{\text {alt }}=S \cap M_{n}(R)_{\text {alt }}$.

\subsection{Symplectic abelian groups}

Define a symplectic abelian group (called a group of type $S$ in [Del01]) to be a finite abelian group $G$ equipped with a nondegenerate alternating pairing 
$[]:, G \times G \rightarrow \mathbb{Q} / \mathbb{Z}$. An isomorphism between two symplectic abelian groups is a group isomorphism that respects the pairings. Let $\operatorname{Sp}(G)$ be the group of automorphisms of $G$ respecting [ , ]. One can show that two symplectic abelian groups are isomorphic if and only if their underlying abelian groups are isomorphic. If $p$ is a prime, define a symplectic $p$-group to be a symplectic abelian group whose order is a power of $p$; in this case, $[$,$] may be viewed$ as taking values in $\mathbb{Q}_{p} / \mathbb{Z}_{p}$.

\subsection{An alternating pairing}

Let $L$ be a free $R$-module of finite rank. Let $\mathcal{A}: L \times L \rightarrow R$ be an alternating $R$-bilinear pairing; alternating means that $\mathcal{A}(x, x)=0$ for all $x \in L$. Define $A: L \rightarrow L^{T}$ to be the induced $R$-homomorphism, sending $x \in L$ to the homomorphism $y \mapsto \mathcal{A}(x, y)$. Let

$$
L^{\perp}:=\left\{x \in L_{K}: \mathcal{A}(x, L) \subseteq R\right\} .
$$

Then $\mathcal{A}_{K}:=\mathcal{A} \otimes K$ induces an alternating pairing

$$
\frac{L^{\perp}}{L} \times \frac{L^{\perp}}{L} \rightarrow \frac{K}{R} .
$$

\subsection{The pairing in the nonsingular case}

Suppose that $A$ is nonsingular in the sense that $A_{K}: L_{K} \rightarrow\left(L^{T}\right)_{K}$ is an isomorphism. Then

$$
\begin{aligned}
L^{\perp} & =\left\{x \in L_{K}: \mathcal{A}(x, L) \subseteq R\right\} \\
& =\left\{x \in L_{K}: A_{K}(x)(L) \subseteq R\right\} \\
& =\left\{x \in L_{K}: A_{K}(x) \in L^{T}\right\} \\
& =A_{K}^{-1} L^{T} .
\end{aligned}
$$

Thus $A_{K}$ induces an isomorphism $L^{\perp} / L \simeq L^{T} / A L=$ coker $A$ of finite torsion $R$-modules. Transporting (2) across this isomorphism yields an alternating pairing

$$
\langle,\rangle_{A}: \text { coker } A \times \operatorname{coker} A \rightarrow \frac{K}{R}
$$

induced by

$$
[,]_{A}: L^{T} \times L^{T} \rightarrow \frac{K}{R}
$$




$$
(x, y) \mapsto \mathcal{A}\left(A_{K}^{-1} x, A_{K}^{-1} y\right)=x \cdot A_{K}^{-1} y
$$

The right kernel of $[,]_{A}$ is

$$
\left\{y: x \cdot A_{K}^{-1} y \in R \text { for all } x \in L^{T}\right\}=\left\{y: A_{K}^{-1} y \in L\right\}=A L .
$$

Since the pairing is alternating, the left kernel is $A L$ too. Thus the left and right kernels of $\langle,\rangle_{A}$ are 0 ; i.e., $\langle,\rangle_{A}$ is nondegenerate.

\subsection{The pairing in the singular case}

Suppose that $A_{K}$ is not an isomorphism. Let $L_{0}=\operatorname{ker} A$. The quotient $L / L_{0}$ is torsion-free, and hence free, since $R$ is a principal ideal domain. Then $\mathcal{A}$ induces a nondegenerate alternating pairing $\mathcal{A}_{1}$ on $L / L_{0}$, corresponding to some $A_{1}: L / L_{0} \rightarrow\left(L / L_{0}\right)^{T}$. We have $\left\{x \in\left(L^{T}\right)_{K}: x . \operatorname{ker} A_{K}=0\right\}=\operatorname{im} A_{K}^{t}=$ $\operatorname{im} A_{K}$ : the first equality holds for any $K$-linear map, while the second holds since $A_{K}$ is alternating. Thus $\left\{x \in L^{T}: x . L_{0}=0\right\}=\left(\operatorname{im} A_{K}\right) \cap L^{T}$; i.e., $\left(L / L_{0}\right)^{T}=(\operatorname{im} A)^{\text {sat }}$. The composition $L \rightarrow L / L_{0} \stackrel{A_{1}}{\rightarrow}\left(L / L_{0}\right)^{T} \hookrightarrow L^{T}$ is $A$, so $\operatorname{im} A_{1}=\operatorname{im} A$ in $L^{T}$ and coker $A_{1} \simeq(\operatorname{im} A)^{\text {sat }} /(\operatorname{im} A) \simeq(\operatorname{coker} A)_{\text {tors }}$. Applying Section 3.4 to $A_{1}$, we obtain an alternating $R$-bilinear pairing

$$
\langle,\rangle_{A}:(\operatorname{coker} A)_{\text {tors }} \times(\operatorname{coker} A)_{\text {tors }} \rightarrow \frac{K}{R}
$$

whose left and right kernels are 0.

\subsection{Lemmas on alternating matrices}

Take $R=\mathbb{Z}_{p}$ and $L=\mathbb{Z}_{p}^{n}$ for some $n \geq 0$; then $L^{T} \simeq \mathbb{Z}_{p}^{n}$ canonically. The homomorphisms $A: L \rightarrow L^{T}$ arising from alternating pairings $\mathcal{A}$ correspond to elements of $M_{n}\left(\mathbb{Z}_{p}\right)_{\text {alt }}$. Given $A \in M_{n}\left(\mathbb{Z}_{p}\right)$, let $\bar{A}:=A \bmod p \in M_{n}\left(\mathbb{F}_{p}\right)$. If $A \in M_{n}\left(\mathbb{Z}_{p}\right)_{\text {alt }}$ and $\operatorname{det} A \neq 0$, then by Section 3.4 , coker $A$ with $\langle,\rangle_{A}$ is a symplectic $p$-group.

Lemma 3.1. Suppose that $A, D \in M_{n}\left(\mathbb{Z}_{p}\right)_{\text {alt }}$, and $\operatorname{det} D \neq 0$. We have $[,]_{A}=[,]_{D}$ if and only if $\operatorname{det} A \neq 0$ and $A^{-1}-D^{-1} \in M_{n}\left(\mathbb{Z}_{p}\right)$.

Proof. This is immediate from (4).

Lemma 3.2. Suppose that $D \in M_{n}\left(\mathbb{Z}_{p}\right)$ and $\operatorname{det} D \neq 0$. Then

$$
\begin{aligned}
& \left\{A \in M_{n}\left(\mathbb{Z}_{p}\right): \operatorname{det} A \neq 0 \text { and } A^{-1}-D^{-1} \in M_{n}\left(\mathbb{Z}_{p}\right)\right\} \\
& =\left\{A \in D+D M_{n}\left(\mathbb{Z}_{p}\right) D: \operatorname{rk} \bar{A}=\operatorname{rk} \bar{D}\right\}
\end{aligned}
$$


Proof. Suppose that $A \in M_{n}\left(\mathbb{Z}_{p}\right)$ is such that $\operatorname{det} A \neq 0$ and $A^{-1}-D^{-1}=N$ for some $N \in M_{n}\left(\mathbb{Z}_{p}\right)$. Multiplying by $A$ on the left yields $I-A D^{-1}=A N$, so $A D^{-1} \in M_{n}\left(\mathbb{Z}_{p}\right)$; similarly $D A^{-1} \in M_{n}\left(\mathbb{Z}_{p}\right)$, so $A D^{-1} \in \mathrm{GL}_{n}\left(\mathbb{Z}_{p}\right)$, and in particular $\operatorname{rk} \bar{D}=\operatorname{rk} \bar{A}$. Multiplying instead by $D$ on the left and $A$ on the right yields $D-A=D N A=D\left(N \cdot A D^{-1}\right) D \in D M_{n}\left(\mathbb{Z}_{p}\right) D$, so $A \in$ $D+D M_{n}\left(\mathbb{Z}_{p}\right) D$.

Conversely, suppose that $A=D+D N D$ with $N \in M_{n}\left(\mathbb{Z}_{p}\right)$, and $\operatorname{rk} \bar{A}=$ $\operatorname{rk} \bar{D}$. Then $\bar{A}=\bar{D}+\bar{D} \bar{N} \bar{D}$, so $\operatorname{ker} \bar{D} \subseteq \operatorname{ker} \bar{A}$, and the rank condition implies $\operatorname{ker} \bar{D}=\operatorname{ker} \bar{A}$. If $v \in \operatorname{ker}(\overline{I+N D})$, then $v \in \operatorname{ker} \bar{A}=\operatorname{ker} \bar{D} ;$ so both $\overline{I+N D}$ and $\overline{N D}$ kill $v$, so $v=0$. Thus $\overline{I+N D} \in \mathrm{GL}_{n}\left(\mathbb{F}_{p}\right)$, so $I+N D \in \mathrm{GL}_{n}\left(\mathbb{Z}_{p}\right)$. Now $D^{-1} A=I+N D$, so its inverse $A^{-1} D$ is in $\operatorname{GL}_{n}\left(\mathbb{Z}_{p}\right)$ too. Multiplying $A=D+D N D$ by $A^{-1}$ on the left and $D^{-1}$ on the right yields $D^{-1}=$ $A^{-1}+A^{-1} D N$, so $A^{-1}-D^{-1}=-\left(A^{-1} D\right) N \in M_{n}\left(\mathbb{Z}_{p}\right)$.

Corollary 3.3. Let $n$ be even, let $e_{1}, \ldots, e_{n / 2} \in \mathbb{Z}_{\geq 0}$, and let

$$
D=\left(\begin{array}{cc}
0 & \operatorname{diag}\left(p^{e_{1}}, \ldots, p^{e_{n / 2}}\right) \\
-\operatorname{diag}\left(p^{e_{1}}, \ldots, p^{e_{n / 2}}\right) & 0
\end{array}\right) \in M_{n}\left(\mathbb{Z}_{p}\right)_{\text {alt }} .
$$

Let $m=2 \#\left\{i \in\{1, \ldots, n / 2\}: e_{i}=0\right\}$. If $A \in M_{n}\left(\mathbb{Z}_{p}\right)_{\text {alt }}$ is chosen at random with respect to Haar measure, then

$$
\operatorname{Prob}\left(\operatorname{det} A \neq 0 \text { and } A^{-1}-D^{-1} \in M_{n}\left(\mathbb{Z}_{p}\right)\right)=\frac{\# \mathrm{GL}_{m}\left(\mathbb{F}_{p}\right)_{\text {alt }}}{\# M_{m}\left(\mathbb{F}_{p}\right)_{\text {alt }}}|\operatorname{det} D|_{p}^{n-1} \text {. }
$$

Proof. Let $e_{n / 2+i}=e_{i}$ for $i=1, \ldots, n / 2$. For $A \in M_{n}\left(\mathbb{Z}_{p}\right)_{\text {alt }}$, the condition $A \in D+D M_{n}\left(\mathbb{Z}_{p}\right) D$ is equivalent to $a_{i j} \equiv d_{i j}\left(\bmod p^{e_{i}} p^{e_{j}} \mathbb{Z}_{p}\right)$ for all $i<j$, so

$$
\operatorname{Prob}\left(A \in D+D M_{n}\left(\mathbb{Z}_{p}\right) D\right)=\prod_{i<j} p^{-e_{i}} p^{-e_{j}}=\prod_{i=1}^{n}\left(p^{-e_{i}}\right)^{n-1}=|\operatorname{det} D|_{p}^{n-1} .
$$

Let $B \in M_{m}\left(\mathbb{Z}_{p}\right)_{\text {alt }}$ be the minor formed by the entries $a_{i j}$ such that $e_{i}=e_{j}=$ 0 . For $A \in D+D M_{n}\left(\mathbb{Z}_{p}\right) D$, the condition $\operatorname{rk} \bar{A}=\operatorname{rk} \bar{D}$ is equivalent to $\bar{B} \in$ $\mathrm{GL}_{m}\left(\mathbb{F}_{p}\right)$, which is independent of the congruences above for $(i, j)$ such that $\left(e_{i}, e_{j}\right) \neq(0,0)$, and which holds with probability $\# \mathrm{GL}_{m}\left(\mathbb{F}_{p}\right)_{\text {alt }} / \# M_{m}\left(\mathbb{F}_{p}\right)_{\text {alt }}$. Multiplying yields the result, by Lemma 3.2.

Combining Corollary 3.3 with Lemma 3.1 yields 
Corollary 3.4. Retain the notation of Corollary 3.3. Then

$$
\operatorname{Prob}\left([,]_{A}=[,]_{D}\right)=\frac{\# \mathrm{GL}_{m}\left(\mathbb{F}_{p}\right)_{\text {alt }}}{\# M_{m}\left(\mathbb{F}_{p}\right)_{\text {alt }}}|\operatorname{det} D|_{p}^{n-1}
$$

Corollary 3.5. Fix any alternating pairing $[]:, \mathbb{Z}_{p}^{n} \times \mathbb{Z}_{p}^{n} \rightarrow \mathbb{Q} / \mathbb{Z}$ inducing a nondegenerate pairing on a finite quotient $G$ of $\mathbb{Z}_{p}^{n}$. Let $m=n-\operatorname{dim}_{\mathbb{F}_{p}} G[p]$. If $A \in M_{n}\left(\mathbb{Z}_{p}\right)_{\text {alt }}$ is chosen at random, then

$$
\operatorname{Prob}\left([,]_{A}=[,]\right)=\frac{\# \mathrm{GL}_{m}\left(\mathbb{F}_{p}\right)_{\text {alt }}}{\# M_{m}\left(\mathbb{F}_{p}\right)_{\text {alt }}}(\# G)^{1-n} .
$$

Proof. The structure theorem for symplectic modules over principal ideal domains implies that there exists a change-of-basis matrix $M \in \mathrm{GL}_{n}\left(\mathbb{Z}_{p}\right)$ and a matrix $D$ as in Corollary 3.3 such that $[]=,[,]_{M^{t} D M}$. The change of basis reduces the statement to Corollary 3.4.

The fraction on the right side of (5) can be evaluated:

Lemma 3.6. For $m \in 2 \mathbb{Z}_{\geq 0}$,

$$
\frac{\# \mathrm{GL}_{m}\left(\mathbb{F}_{p}\right)_{\text {alt }}}{\# M_{m}\left(\mathbb{F}_{p}\right)_{\text {alt }}}=\prod_{i=1}^{m / 2}\left(1-p^{1-2 i}\right)
$$

Proof. There are $p^{m-1}-1$ possibilities for the first column of a matrix in $\mathrm{GL}_{m}\left(\mathbb{F}_{p}\right)_{\text {alt }}$, and the number of choices for the rest of the matrix is independent of this first choice, as one sees by performing a change of basis of $\mathbb{F}_{p}^{n}$ fixing $(1,0, \ldots, 0)^{t}$. If the first column is $(0,1,0,0, \ldots, 0)^{t}$, there are $p^{m-2}$ possibilities for the second column (it has the shape $(1,0, *, \cdots, *)$ ), and then the lower $(m-2) \times(m-2)$ block is an arbitrary element of $\mathrm{GL}_{m-2}\left(\mathbb{F}_{p}\right)_{\text {alt }}$. Thus

$$
\# \mathrm{GL}_{m}\left(\mathbb{F}_{p}\right)_{\text {alt }}=\left(p^{m-1}-1\right) p^{m-2} \# \mathrm{GL}_{m-2}\left(\mathbb{F}_{p}\right)_{\text {alt }}
$$

Induction on $m$ yields $\# \mathrm{GL}_{m}\left(\mathbb{F}_{p}\right)_{\text {alt }}$, and we divide by $\# M_{m}\left(\mathbb{F}_{p}\right)_{\text {alt }}=p^{\left(\begin{array}{c}m \\ 2\end{array}\right)}$.

The following will be used in Section 5.4.

Lemma 3.7. The probability that a random $A \in M_{n}\left(\mathbb{F}_{p}\right)_{\text {alt }}$ satisfies the inequality $\operatorname{dim} \operatorname{ker} A \geq n / 2$ tends to 0 as $n \rightarrow \infty$. 
Proof. Let $k=\lceil n / 2\rceil$. The number of $A \in M_{n}\left(\mathbb{F}_{p}\right)_{\text {alt }}$ with $\operatorname{dim} \operatorname{ker} A \geq$ $n / 2$ is at most the number of pairs $(A, K)$ with $A \in M_{n}\left(\mathbb{F}_{p}\right)_{\text {alt }}$ and $K$ a $k$-dimensional subspace of ker $A$. The number of $K$ 's is $O\left(p^{k(n-k)}\right)$. For each $K$, the $A$ 's vanishing on $K$ correspond to alternating maps from the $(n-k)$ dimensional space $\mathbb{F}_{p}^{n} / K$ to its dual, of which there are $p^{(n-k)(n-k-1) / 2}$. Thus the total number of $A$ with $\operatorname{dim} \operatorname{ker} A \geq n / 2$ is at most

$$
O\left(p^{k(n-k)}\right) \cdot p^{(n-k)(n-k-1) / 2}=O\left(p^{(n-k)(n+k-1) / 2}\right) .
$$

Dividing by $\# M_{n}\left(\mathbb{F}_{p}\right)_{\text {alt }}=p^{n(n-1) / 2}$ yields $O\left(p^{-k(k-1) / 2}\right)$, which tends to 0 as $n \rightarrow \infty$.

Remark 3.8. In fact, Lemma 3.7 remains true if $n / 2$ is replaced by any function of $n$ tending to $\infty$, but the $n / 2$ version suffices for our application.

\subsection{The distribution in the nonsingular case}

The following theorem states that the limit $\mathscr{A}_{0}$ in Theorem 1.10(a) exists, and provides an explicit formula for its value:

Theorem 3.9. For each symplectic p-group $G$, if $A$ is chosen at random in $M_{n}\left(\mathbb{Z}_{p}\right)_{\text {alt }}$ with respect to Haar measure for even $n$, then

$$
\lim _{\substack{n \rightarrow \infty \\ n \text { even }}} \operatorname{Prob}(\operatorname{coker} A \simeq G)=\frac{\# G}{\# \operatorname{Sp}(G)} \prod_{i=1}^{\infty}\left(1-p^{1-2 i}\right) .
$$

Moreover, the sum of the right side over all such $G$ equals 1 .

Proof. Define

$$
\pi_{n}(G):=\operatorname{Prob}(\operatorname{coker} A \simeq G), \quad \text { and } \quad \pi(G):=\lim _{\substack{n \rightarrow \infty \\ n \text { even }}} \pi_{n}(G) .
$$

Let $m=n-\operatorname{dim}_{\mathbb{F}_{p}} G[p] \in 2 \mathbb{Z}_{\geq 0}$. Given a surjection $f: \mathbb{Z}_{p}^{n} \rightarrow G$, we may pull back the pairing on $G$ to obtain an alternating pairing on $\mathbb{Z}_{p}^{n}$. This defines a surjection from $\operatorname{Surj}\left(\mathbb{Z}_{p}^{n}, G\right)$ to the set of alternating pairings [ , ]: $\mathbb{Z}_{p}^{n} \times \mathbb{Z}_{p}^{n} \rightarrow$ $\mathbb{Q} / \mathbb{Z}$ such that the induced $S$-group is isomorphic to $G$. Each fiber is the orbit of a free action of $\operatorname{Sp}(G)$ on $\operatorname{Surj}\left(\mathbb{Z}_{p}^{n}, G\right)$ (by post-composition), so the number of such [ , ]'s is \# $\operatorname{Surj}\left(\mathbb{Z}_{p}^{n}, G\right) / \# \operatorname{Sp}(G)$. By Corollary 3.5, the probability that $[,]_{A}$ equals any fixed one of these $[$,$] equals$

$$
\frac{\# \mathrm{GL}_{m}\left(\mathbb{F}_{p}\right)_{\mathrm{alt}}}{\# M_{m}\left(\mathbb{F}_{p}\right)_{\text {alt }}}(\# G)^{1-n} .
$$


Multiplying yields

$$
\pi_{n}(G)=\frac{\# \operatorname{Surj}\left(\mathbb{Z}_{p}^{n}, G\right)}{\# \operatorname{Sp}(G)} \frac{\# \mathrm{GL}_{m}\left(\mathbb{F}_{p}\right)_{\text {alt }}}{\# M_{m}\left(\mathbb{F}_{p}\right)_{\text {alt }}}(\# G)^{1-n} .
$$

As $n \rightarrow \infty$, we have $m \rightarrow \infty$ through even integers, and we also have $\# \operatorname{Surj}\left(\mathbb{Z}_{p}^{n}, G\right) /(\# G)^{n} \rightarrow 1$ since almost all homomorphisms $\mathbb{Z}_{p}^{n} \rightarrow G$ are surjective, so by Lemma 3.6, we obtain

$$
\pi(G)=\frac{\# G}{\# \operatorname{Sp}(G)} \prod_{i=1}^{\infty}\left(1-p^{1-2 i}\right) .
$$

It remains to prove that $\sum_{G} \pi(G)=1$. For fixed $n$, the event that coker $A$ is infinite corresponds to the $\mathbb{Z}_{p}$-points of a hypersurface in the affine space of alternating matrices, so Proposition 2.1(b) shows that it has probability 0; thus $\sum_{G} \pi_{n}(G)=1$. By Fatou's lemma, $\sum_{G} \pi(G) \leq 1$. In particular,

$$
\sum_{G} \frac{\# G}{\# \operatorname{Sp}(G)} \leq \prod_{i=1}^{\infty}\left(1-p^{1-2 i}\right)^{-1}<\infty
$$

In (6), we have $\# \operatorname{Surj}\left(\mathbb{Z}_{p}^{n}, G\right) \leq \# G^{n}$ and $\# \mathrm{GL}_{m}\left(\mathbb{F}_{p}\right)_{\text {alt }} \leq \# M_{m}\left(\mathbb{F}_{p}\right)_{\text {alt }}$, so $\pi_{n}(G) \leq \# G / \# \operatorname{Sp}(G)$; this lets us apply the dominated convergence theorem to deduce

$$
\sum_{G} \pi(G)=\sum_{G} \lim \pi_{n}(G)=\lim \sum_{G} \pi_{n}(G)=1 .
$$

\subsection{The distribution in the singular case}

Fix $n \geq 0$. The variety $\mathbb{A}^{n(n-1) / 2}$ parametrizing alternating $n \times n$ matrices over any field can be stratified according to the rank of the matrix. Namely, given an integer $r$ with $0 \leq r \leq n$ and $n-r$ even, let $V_{n, r}$ be the locally closed subvariety parametrizing alternating $n \times n$ matrices of rank $n-r$. (The hypotheses on $r$ are needed to ensure that $V_{n, r}$ is nonempty.) The existence of symplectic bases shows that $V_{n, r}$ is a homogeneous space for the action of $\mathrm{GL}_{n}$ on $\mathbb{A}^{n(n-1) / 2}$.

Lemma 3.10. $\operatorname{dim} V_{n, r}=\left(\begin{array}{l}n \\ 2\end{array}\right)-\left(\begin{array}{l}r \\ 2\end{array}\right)$.

Proof. We may work over an algebraically closed field $k$. There is a morphism from $V_{n, r}$ to the Grassmannian of $(n-r)$-planes $\Pi$ in $n$-space, sending a matrix $A \in M_{n}(k)$ to $\operatorname{im}(A)$. The fiber above $\Pi$ parametrizes nondegenerate 
alternating maps from $k^{n} /(\operatorname{im} A)^{\perp} \simeq \operatorname{im}(A)^{T}$ to $\operatorname{im}(A)$, so each fiber has dimension $\left(\begin{array}{c}n-r \\ 2\end{array}\right)$. Thus $\operatorname{dim} V_{n, r}=r(n-r)+\left(\begin{array}{c}n-r \\ 2\end{array}\right)=\left(\begin{array}{c}n \\ 2\end{array}\right)-\left(\begin{array}{c}r \\ 2\end{array}\right)$.

Alternatively, one could compute the dimension of the stabilizer of

$$
\left(\begin{array}{ccc} 
& I_{(n-r) / 2} & \\
-I_{(n-r) / 2} & & 0_{r}
\end{array}\right) \in V_{n, r}
$$

by writing an equation in $2 \times 2$ block matrices with blocks of size $n-r$ and $r$.

We have the locally closed stratification

$$
\mathbb{A}^{n(n-1) / 2}=\bigcup_{r} V_{n, r},
$$

where $r$ ranges over integers with $0 \leq r \leq n$ and $n-r$ even. The Zariski closure $\bar{V}_{n, r}$ of $V_{n, r}$ in $\mathbb{A}^{n(n-1) / 2}$ is the locus $\bigcup_{s \geq r} V_{n, s}$ of alternating matrices of rank at most $n-r$ : this is closed since it is cut out by the vanishing of the $(n-r+1) \times(n-r+1)$ minors, and it is in the closure of $V_{n, r}$, as one can see from using standard symplectic matrices. We may extend $\bar{V}_{n, r}$ to a closed subscheme of $\mathbb{A}_{\mathbb{Z}_{n}}^{n(n-1) / 2}$ defined by the vanishing of the same minors.

Let $\mathcal{A}_{n, r}=V_{n, r}\left(\mathbb{Q}_{p}\right) \cap M_{n}\left(\mathbb{Z}_{p}\right)_{\text {alt }}$. (Although $V_{n, r}$ can naturally be extended to a $\mathbb{Z}_{p}$-scheme, $\mathcal{A}_{n, r}$ is generally larger than $V_{n, r}\left(\mathbb{Z}_{p}\right)$, since the latter consists of the $A \in V_{n, r}\left(\mathbb{Q}_{p}\right) \cap M_{n}\left(\mathbb{Z}_{p}\right)_{\text {alt }}$ satisfying the extra condition that the $\bmod p$ reduction has the same rank as $A$, namely $r$.) Then we have an analogous locally closed stratification of topological spaces

$$
M_{n}\left(\mathbb{Z}_{p}\right)_{\text {alt }}=\bigcup_{r} \mathcal{A}_{n, r}
$$

The closure $\overline{\mathcal{A}}_{n, r}$ of $\mathcal{A}_{n, r}$ equals $\bar{V}_{n, r}\left(\mathbb{Z}_{p}\right)=\bigcup_{s \geq r} \mathcal{A}_{n, s}$.

Fix $r$. Proposition 2.1 and Corollary 2.2 applied to $X=\bar{V}_{n, r}$ yields measures $\mu$ and $\nu$ on $\overline{\mathcal{A}}_{n, r}$. By Proposition 2.1(b), $\mu\left(\overline{\mathcal{A}}_{n, s}\right)=0$ for $s>r$, so the probability measure $\nu$ restricts to a probability measure $\nu$ on the open subset $\mathcal{A}_{n, r}$. We use $\mu$ to denote the $\mu$ for different varieties; the meaning will be clear from context.

The following generalization of Theorem 3.9 states that the limit $\mathscr{A}_{r}$ in Theorem 1.10(a) exists for each $r \in \mathbb{Z}_{\geq 0}$ and gives an explicit formula for its value. In particular, it implies Theorem 1.10(a).

Theorem 3.11. Fix $r \in \mathbb{Z}_{\geq 0}$, and fix a symplectic p-group $G$. If $A \in \mathcal{A}_{n, r}$ is chosen at random with respect to $\nu$, then 


$$
\lim _{\substack{n \rightarrow \infty \\ n-r \text { even }}} \operatorname{Prob}\left((\operatorname{coker} A)_{\text {tors }} \simeq G\right)=\frac{(\# G)^{1-r}}{\# \operatorname{Sp}(G)} \prod_{i=r+1}^{\infty}\left(1-p^{1-2 i}\right) .
$$

Moreover, the sum of the right side over all such $G$ equals 1.

To prove Theorem 3.11, we need two lemmas. Let $|\operatorname{det}|^{s}: M_{n}\left(\mathbb{Z}_{p}\right) \rightarrow \mathbb{R}$ be the function $A \mapsto|\operatorname{det} A|_{p}^{s}$.

Lemma 3.12. For any $s \in \mathbb{R}_{\geq 0}$, we have

$$
\int_{M_{n}\left(\mathbb{Z}_{p}\right)_{\text {alt }}}|\operatorname{det}|^{s} \mu=\prod_{i=1}^{n / 2} \frac{1-p^{1-2 i}}{1-p^{1-2 i-2 s}} .
$$

Proof. The proof is an easy induction on $n$ : see [Igu00, p. 164].

Lemma 3.13. Define

$$
\begin{aligned}
\beta: \mathrm{GL}_{n}\left(\mathbb{Z}_{p}\right) \times \mathcal{A}_{n-r, 0} & \longrightarrow \mathcal{A}_{n, r} \\
(M, A) & \longmapsto M^{t}\left(\begin{array}{cc}
A & 0 \\
0 & 0
\end{array}\right) M .
\end{aligned}
$$

Then $\beta_{*}\left(\mu \times|\operatorname{det}|^{r} \mu\right)=c \mu$ for some $c>0$ depending on $n$ and $r$.

Proof. Given $B \in M_{n}\left(\mathbb{Z} / p^{e} \mathbb{Z}\right)$ in the reduction of $\mathcal{A}_{n, r}$, we must count the number of $(M, A) \in \mathrm{GL}_{n}\left(\mathbb{Z} / p^{e} \mathbb{Z}\right) \times M_{n-r}\left(\mathbb{Z} / p^{e} \mathbb{Z}\right)_{\text {alt }}$ such that

$$
M^{t}\left(\begin{array}{cc}
A & 0 \\
0 & 0
\end{array}\right) M=B
$$

We may assume that $e$ is large enough that some $(n-r) \times(n-r)$ minor of $B$ has nonzero determinant $\bmod p^{e}$. We may assume also that $B$ itself is of the form $\left(\begin{array}{ll}C & 0 \\ 0 & 0\end{array}\right)$; then the set of $(M, A)$ is

$$
\left\{\left(N^{-1}, N^{t} B N\right): N \in \mathrm{GL}_{n}\left(\mathbb{Z} / p^{e} \mathbb{Z}\right), N^{t} B N \text { has the form }\left(\begin{array}{ll}
* & 0 \\
0 & 0
\end{array}\right)\right\} \text {. }
$$

If $N=\left(\begin{array}{ll}P & Q \\ R & S\end{array}\right)$, then the condition on $N$ is equivalent to $P \in \mathrm{GL}_{n-r}\left(\mathbb{Z} / p^{e} \mathbb{Z}\right)$, $P^{t} C Q=0, Q^{t} C P=0$, and $Q^{t} C Q=0$ (invertibility of $P$ follows from comparing determinants of minors of $B$ to those of $\left.N^{t} B N\right)$. Since $C^{t}=-C$, these conditions are equivalent to $P \in \mathrm{GL}_{n-r}\left(\mathbb{Z} / p^{e} \mathbb{Z}\right)$ and $C Q=0$. The 
number of possibilities for $P$ is $\# \mathrm{GL}_{n-r}\left(\mathbb{Z} / p^{e} \mathbb{Z}\right)$, which is independent of $B$. On the other hand, if we view $C$ as a map from the finite group $\left(\mathbb{Z} / p^{e} \mathbb{Z}\right)^{n-r}$ to itself, then its kernel has the same size as its cokernel, which is $|\operatorname{det} C|_{p}^{-1}$, so the number of possibilities for $Q$ is $|\operatorname{det} C|_{p}^{-r}$. Thus if each pair $(M, A)$ is weighted by $|\operatorname{det} A|_{p}^{r}=|\operatorname{det} C|_{p}^{r}$, then the weighted count of such pairs is independent of $B$.

Proof of Theorem 3.11. Define

$$
\mathcal{A}_{n, r}(G):=\left\{A \in \mathcal{A}_{n, r}:(\operatorname{coker} A)_{\mathrm{tors}} \simeq G\right\} .
$$

As $n \rightarrow \infty$ through integers with $n-r$ even,

$$
\begin{aligned}
\nu\left(\mathcal{A}_{n, r}(G)\right) & =\frac{\int_{\mathcal{A}_{n, r}(G)} \mu}{\int_{\mathcal{A}_{n, r}} \mu} \\
& =\frac{\int_{\mathrm{GL}_{n}\left(\mathbb{Z}_{p}\right)} \mu \cdot \int_{\mathcal{A}_{n-r, 0}(G)}|\operatorname{det}|^{r} \mu}{\int_{\mathrm{GL}_{n}\left(\mathbb{Z}_{p}\right)} \mu \cdot \int_{\mathcal{A}_{n-r, 0}}|\operatorname{det}|^{r} \mu} \quad \text { (by Lemma 3.13) } \\
& =\frac{\# G^{-r} \int_{\mathcal{A}_{n-r, 0}(G)} \mu}{\int_{\mathcal{A}_{n-r, 0}}|\operatorname{det}|^{r} \mu} \\
& \rightarrow \frac{\# G^{-r} \frac{\# G}{\# \operatorname{Sp}(G)} \prod_{i=1}^{\infty}\left(1-p^{1-2 i}\right)}{\prod_{i=1}^{\infty} \frac{1-p^{1-2 i}}{1-p^{1-2 i-2 r}}}
\end{aligned}
$$

(by Theorem 3.9 and Lemma 3.12)

$$
=\frac{\# G^{1-r}}{\# \operatorname{Sp}(G)} \prod_{i=r+1}^{\infty}\left(1-p^{1-2 i}\right) .
$$

The same argument as in the proof of Theorem 3.9 shows that these numbers sum to 1 .

\section{Orthogonal Grassmannians}

\subsection{Grassmannians}

Given $0 \leq m \leq n$, for each commutative ring $R$ let $\operatorname{Gr}_{m, n}(R)$ be the set of direct summands $W$ of $R^{n}$ that are locally free of rank $m$. This functor is represented by a smooth projective scheme $\mathrm{Gr}_{m, n}$ of relative dimension $m(n-m)$ over $\mathbb{Z}$, called a Grassmannian: see [EGA I, §9.7 and §9.8]. (The definition of $\mathrm{Gr}_{m, n}$ there is in terms of locally free quotients, but this is equivalent: a locally free quotient of constant rank is projective [Bou98, II.§5.2, 
Theorem 1], so the kernel of the quotient map is a locally free direct summand; [Bou98, II.§5.2, Theorem 1] shows also that it does not matter whether we interpret "locally free" stalkwise or on an open cover of $\operatorname{Spec} R$.)

\subsection{Maximal isotropic direct summands}

Now equip $R^{2 n}$ with the hyperbolic quadratic form $Q: R^{2 n} \rightarrow R$ given by

$$
Q\left(x_{1}, \ldots, x_{n}, y_{1}, \ldots, y_{n}\right):=\sum_{i=1}^{n} x_{i} y_{i} .
$$

The associated bilinear pairing is $\langle a, b\rangle:=Q(a+b)-Q(a)-Q(b)$. A direct summand $Z$ is called isotropic if $\left.Q\right|_{Z}=0$ (in general this is stronger than requiring that $\left.\left.\langle\rangle\right|_{,Z \times Z}=0\right)$. Let $\mathrm{OGr}_{n}(R)$ be the set of isotropic $Z \in$ $\operatorname{Gr}_{n, 2 n}(R)$. Such $Z$ will also be called maximal isotropic direct summands of $R^{2 n}$.

Lemma 4.1. Let $R$ be a ring. Let $X, X^{\prime} \in \operatorname{OGr}_{n}(R)$ be modules such that $X \oplus X^{\prime} \rightarrow R^{2 n}$ is an isomorphism.

(a) The restriction of $\langle$,$\rangle to X \times X^{\prime}$ identifies $X^{\prime}$ with $X^{T}$.

(b) Let $\phi: X \rightarrow X^{\prime}$ be an $R$-module homomorphism. Then $\operatorname{graph}(\phi) \in$ $\mathrm{OGr}_{n}(R)$ if and only if $\phi$ is alternating (with respect to the identification above).

Proof.

(a) By tensoring with $R / \mathfrak{m}$ for every maximal ideal $\mathfrak{m} \subseteq R$, we reduce to the case in which $R$ is a field. The kernel of $X^{\prime} \rightarrow X^{T}$ is orthogonal to $X$, but also to $X^{\prime}$ since $X^{\prime}$ is isotropic. By nondegeneracy of $\langle$,$\rangle on$ $R^{2 n}$, this kernel is 0 . Since $X^{\prime}$ and $X^{T}$ are vector spaces of the same dimension, $X^{\prime} \rightarrow X^{T}$ is an isomorphism.

(b) For $x \in X$,

$$
\langle x, \phi(x)\rangle=Q(x+\phi(x))-Q(x)-Q(\phi(x))=Q(x+\phi(x)) .
$$

By definition, $\phi$ is alternating if and only if the left side is 0 for all $x$. Also by definition, $\operatorname{graph}(\phi) \in \mathrm{OGr}_{n}(R)$ if and only if the right side is 0 for all $x \in X$.

Proposition 4.2. Let $\mathrm{O}_{2 n}$ be the orthogonal group of $\left(\mathbb{Z}^{2 n}, Q\right)$.

(a) Let $R$ be a field, a discrete valuation ring, or a quotient thereof. The action of $\mathrm{O}_{2 n}(R)$ on $\mathrm{OGr}_{n}(R)$ is transitive. 
(b) Let $k$ be a field. For each $m \in\{0,1, \ldots, n\}$, the action of $\mathrm{O}_{2 n}(k)$ on $\left\{(Y, Z) \in \mathrm{OGr}_{n}(k)^{2}: \operatorname{dim}(Y \cap Z)=m\right\}$ is transitive.

Proof.

(a) The hypothesis on $R$ implies that every direct summand of $R^{2 n}$ is free. Let $Z \in \operatorname{OGr}_{n}(R)$. Choose a basis $z_{1}, \ldots, z_{n}$ of $Z$. Choose a basis $y_{1}, \ldots, y_{n}$ for an $R$-module complement $Y$ of $Z$ in $R^{2 n}$. Since $\langle$,$\rangle is nondegen-$ erate, we can change the basis of $Y$ to assume that $\left\langle y_{i}, z_{j}\right\rangle=\delta_{i j}$. Let $y_{i}^{\prime}:=y_{i}-Q\left(y_{i}\right) z_{i}-\sum_{j>i}\left\langle y_{i}, y_{j}\right\rangle z_{j}$. Then the $R$-linear map sending the standard basis of $R^{2 n}$ to $z_{1}, \ldots, z_{n}, y_{1}^{\prime}, \ldots, y_{n}^{\prime}$ is an element of $\mathrm{O}_{2 n}(R)$ sending $W$ to $Z$.

(b) Given a pair $(Y, Z)$ in the set, choose a basis $x_{1}, \ldots, x_{m}$ for $Y \cap Z$, extend it to bases $x_{1}, \ldots, x_{m}, z_{m+1}, \ldots, z_{n}$ of $Z$ and $x_{1}, \ldots, x_{m}, y_{m+1}, \ldots, y_{n}$ of $Y$, and replace $y_{m+1}, \ldots, y_{n}$ by linear combinations so that $\left\langle y_{i}, z_{j}\right\rangle=\delta_{i j}$ for $i, j \in[m+1, n]$. Inductively choose $w_{i} \in k^{2 n}$ for $i=1, \ldots, m$ so that $w_{i}$ is orthogonal to the $w_{j}$ for $j<i$ and to all the $x_{j}, y_{j}, z_{j}$ except $\left\langle w_{i}, x_{i}\right\rangle=1$. Adjust each $w_{i}$ by a multiple of $x_{i}$ in order to assume in addition that $Q\left(w_{i}\right)=0$.

Now, given another pair $\left(Y^{\prime}, Z^{\prime}\right)$ in the set, the $R$-linear map sending the $w_{i}, x_{i}, y_{i}, z_{i}$ to their counterparts is an element of $\mathrm{O}_{2 n}(R)$ sending $(Y, Z)$ to $\left(Y^{\prime}, Z^{\prime}\right)$.

Let $W$ be the maximal isotropic direct summand $\mathbb{Z}^{n} \times 0$ of $\mathbb{Z}^{2 n}$.

Lemma 4.3. Let $\bar{W} \in \mathrm{OGr}_{n}\left(\mathbb{F}_{p}\right)$ be the mod $p$ reduction of $W$. Let $Y \subseteq \bar{W}$ be an $\mathbb{F}_{p}$-subspace. Then the subgroup of $\mathrm{O}_{2 n}\left(\mathbb{Z}_{p}\right)$ preserving $W_{\mathbb{Z}_{p}}$ and $Y$ acts transitively on $\left\{X \in \mathrm{OGr}_{n}\left(\mathbb{F}_{p}\right): X \cap \bar{W}=Y\right\}$.

Proof. For any $X, X^{\prime}$ in the set, Proposition 4.2(b) yields an element $\bar{\alpha} \in$ $\mathrm{O}_{2 n}\left(\mathbb{F}_{p}\right)$ sending $(\bar{W}, X)$ to $\left(\bar{W}, X^{\prime}\right)$; then $\bar{\alpha} \in \operatorname{Stab}_{\mathrm{O}_{2 n}\left(\mathbb{F}_{p}\right)}(\bar{W})$. It remains to lift $\bar{\alpha}$ to an element $\alpha \in \operatorname{Stab}_{\mathrm{O}_{2 n}\left(\mathbb{Z}_{p}\right)}(W)$, since such an $\alpha$ will preserve also $X \cap \bar{W}=X^{\prime} \cap \bar{W}=Y$. By Hensel's lemma, the lift exists if the stabilizer group scheme $S \leq \mathrm{O}_{2 n}$ of $W$ is smooth over $\mathbb{Z}$. In fact, if we define $W^{\prime}:=$ $0 \times \mathbb{Z}^{n} \in \mathrm{OGr}_{n}(\mathbb{Z})$, then there is a short exact sequence of group schemes

$$
1 \rightarrow B \rightarrow S \rightarrow \mathrm{GL}_{W} \rightarrow 1
$$

where $B$ is the additive group scheme of alternating maps $\beta: W^{\prime} \rightarrow W$; namely, $\beta \in B$ maps to the unique $s \in S$ such that $s\left(w^{\prime}\right)=w^{\prime}+\beta\left(w^{\prime}\right)$ for all $w^{\prime} \in W^{\prime}$, and $S \rightarrow \mathrm{GL}_{W}$ is defined by the action of $S$ on $W$. Since $B$ and $\mathrm{GL}_{W}$ are smooth, so is $S$. 


\subsection{Orthogonal Grassmannians as schemes}

Proposition 4.4. For each $n \geq 0$, the functor $\mathrm{OGr}_{n}$ is represented by a smooth projective scheme of relative dimension $n(n-1) / 2$ over $\mathbb{Z}$, called an orthogonal Grassmannian.

Proof. See [SGA 7II XII, Proposition 2.8], where $\mathrm{OGr}_{n}$ is denoted Gén $(X)$. The expression for the relative dimension arises in the proof there as the rank of $\bigwedge^{2} W$.

If $V \simeq R^{2 n}$ for some ring $R$, also write $\mathrm{OGr}_{V}$ for the $R$-scheme $\mathrm{OGr}_{n, R}$. Proposition 4.5. Fix $n>0$.

(a) The scheme $\mathrm{OGr}_{n}$ is a disjoint union of two isomorphic schemes $\mathrm{OGr}_{n}^{\text {even }}$ and $\mathrm{OGr}_{n}^{\text {odd }}$, distinguished by the property that for $Z \in \mathrm{OGr}_{n}(k)$ for a field $k$,

$$
Z \in \operatorname{OGr}_{n}^{\text {even }}(k) \Longleftrightarrow \operatorname{dim}\left(Z \cap W_{k}\right) \text { is even. }
$$

(b) If $k$ is a field, then $\mathrm{OGr}_{n, k}^{\mathrm{even}}$ and $\mathrm{OGr}_{n, k}^{\text {odd }}$ are geometrically integral.

(c) For any field $k$, two points $Z, Z^{\prime} \in \mathrm{OGr}_{n}(k)$ belong to the same component of $\mathrm{OGr}_{n, k}$ if and only if $\operatorname{dim}\left(Z \cap Z^{\prime}\right) \equiv n(\bmod 2)$.

Proof. See the proof of [SGA 7II, XII, Proposition 2.8], which shows that there is a morphism $e: \operatorname{OGr}_{n} \rightarrow \operatorname{Spec}(\mathbb{Z} \times \mathbb{Z})$ with geometrically connected fibers. Define $\mathrm{OGr}_{n}^{\text {even }}$ and $\mathrm{OGr}_{n}^{\text {odd }}$ as the preimages of the components of $\operatorname{Spec}(\mathbb{Z} \times \mathbb{Z})$; they can be chosen so that both (8) and (c) hold, by [SGA $7_{\mathrm{II}}$, XII, Proposition 1.12]. Geometrically connected and smooth imply geometrically integral.

Remark 4.6. For $n=0$, we may define $\mathrm{OGr}_{0}^{\text {even }}:=\mathrm{OGr}_{0}$ and $\mathrm{OGr}_{0}^{\text {odd }}:=\emptyset$.

Corollary 4.7. If $Z_{1}, Z_{2}, Z_{3} \in \mathrm{OGr}_{n}(k)$ for a field $k$, then

$$
\operatorname{dim}\left(Z_{1} \cap Z_{2}\right)+\operatorname{dim}\left(Z_{2} \cap Z_{3}\right)+\operatorname{dim}\left(Z_{3} \cap Z_{1}\right) \equiv n \quad(\bmod 2) .
$$

Proof. By Proposition 4.5(c), the parity of $\operatorname{dim}\left(Z_{1} \cap Z_{2}\right)-n$ measures whether $Z_{1}$ and $Z_{2}$ belong to the same component. Summing three such integers gives the parity of the number of component switches in hopping from $Z_{1}$ to $Z_{2}$ to $Z_{3}$ and back to $Z_{1}$; the latter number is even.

Lemma 4.8. Let $q=p^{e}$ for a prime $p$ and $e \geq 1$. Then

$$
\# \mathrm{OGr}_{n}(\mathbb{Z} / q \mathbb{Z})=q^{n(n-1) / 2} \prod_{i=1}^{n}\left(1+p^{i-n}\right)
$$


Proof. The case $e=1$ is [PR12, Proposition 2.6(b)]. The $e=1$ case implies the general case since $\mathrm{OGr}_{n}$ is smooth of relative dimension $n(n-1) / 2$.

\subsection{Schubert subschemes}

Suppose that $0 \leq r \leq n$. For a field $k$, let $\mathcal{S}_{n, r}(k)$ be the set of spaces $Z \in \operatorname{OGr}_{n}^{\text {parity }(r)}(k)$ such that $\operatorname{dim}\left(Z \cap W_{k}\right) \geq r$, or equivalently, the set of $Z \in \mathrm{OGr}_{n}(k)$ such that $\operatorname{dim}\left(Z \cap W_{k}\right)-r \in 2 \mathbb{Z}_{\geq 0}$. For an arbitrary ring $R$, let $\mathcal{S}_{n, r}(R)$ be the set of $Z \in \mathrm{OGr}_{n}(R)$ such that $Z_{k} \in \mathcal{S}_{n, r}(k)$ for every field $k$ that is a quotient of $R$.

Proposition 4.9. For $0 \leq r \leq n$, the functor $\mathcal{S}_{n, r}$ is represented by a closed subscheme of $\mathrm{OGr}_{n}$ whose fibers over $\mathbb{Z}$ have dimension

$$
n(n-1) / 2-r(r-1) / 2=(n-r)(n+r-1) / 2 .
$$

Proof. There is a closed subscheme of $\mathrm{Gr}_{n, 2 n}$ whose $k$-points parametrize $n$-dimensional subspaces $Z$ with $\operatorname{dim}(Z \cap W) \geq r$. Its intersection with the closed subscheme $\operatorname{OGr}_{n}^{\text {parity }(r)}$ is $\mathcal{S}_{n, r}$.

To compute the fiber dimension, we work over a field $k$, and consider the closed subscheme $\mathcal{S}_{n, r}^{\prime} \subseteq \mathcal{S}_{n, r} \times \mathrm{Gr}_{r, n}$ parametrizing pairs $(Z, X)$ such that $X \subseteq Z \cap W$. Given $X \subseteq W$, the quadratic form $Q$ restricts to a hyperbolic quadratic form on $X^{\perp} / X$, and the $Z$ 's containing $X$ are in bijection with the maximal isotropic subspaces of $X^{\perp} / X$, via $Z \mapsto Z / X$. Thus the second projection $\mathcal{S}_{n, r}^{\prime} \rightarrow \mathrm{Gr}_{r, n}$ has fibers isomorphic to $\mathrm{OGr}_{n-r}$, so

$$
\begin{aligned}
\operatorname{dim} \mathcal{S}_{n, r}^{\prime} & =\operatorname{dim} \mathrm{Gr}_{r, n}+\operatorname{dim} \mathrm{OGr}_{n-r} \\
& =r(n-r)+(n-r)(n-r-1) / 2 \\
& =(n-r)(n+r-1) / 2 .
\end{aligned}
$$

On the other hand, there is an open subscheme $\mathcal{S}_{n, r}^{\circ} \subseteq \mathcal{S}_{n, r}$ above which $\mathcal{S}_{n, r}^{\prime} \rightarrow \mathcal{S}_{n, r}$ is an isomorphism, namely the subscheme parametrizing $Z$ for which $\operatorname{dim}(Z \cap W)$ equals $r$. If we view $\mathcal{S}_{n, r}^{\circ}$ as an open subscheme of $\mathcal{S}_{n, r}^{\prime}$, which maps to $\mathrm{Gr}_{r, n}$, then its fiber above $X$ is the open subscheme of $\mathrm{OGr}_{X^{\perp} / X}$ consisting of subspaces $Y$ not meeting $W / X$, and those subspaces are exactly the graphs of alternating maps from an $(n-r)$-dimensional space to its dual, so the fiber is $\mathbb{A}^{(n-r)(n-r-1) / 2}$. It follows that $\mathcal{S}_{n, r}^{\circ}$ has the same dimension as $\mathcal{S}_{n, r}^{\prime}$. Since $\mathcal{S}_{n, r}$ is sandwiched in between, it too has the same dimension.

Call $\mathcal{S}_{n, r}$ a Schubert subscheme. It could also have been defined as the closure of the locally closed subscheme $\mathcal{S}_{n, r}^{\circ} \subseteq \mathrm{OGr}_{n}$. 


\section{Modeling Selmer groups using maximal isotropic submodules}

\subsection{Properties of the short exact sequence}

Let $Z$ and $W$ be maximal isotropic direct summands of $V$ as in Section 1.2. (For the time being, they do not need to be random; what we say here applies to any choice of $Z$ and $W$.) From $Z$ and $W$ construct

$$
0 \rightarrow R \rightarrow S \rightarrow T \rightarrow 0
$$

as in Section 1.2.

Proposition 5.1. The maximal divisible subgroup of $S$ is $R$.

Proof. Since the group $R=(Z \cap W) \otimes \frac{\mathbb{Q}_{p}}{\mathbb{Z}_{p}}$ is divisible, it suffices to show that every infinitely divisible element $a$ of $S$ is in $R$. Suppose that $a \in S$ is infinitely divisible. For each $m \geq 1$, write $a=p^{m} a_{m}$ for some $a_{m} \in S$. By definition of $S$, we have $a_{m}=\left(z_{m} \bmod V\right)=\left(w_{m} \bmod V\right)$ for some $z_{m} \in Z \otimes \mathbb{Q}_{p}$ and $w_{m} \in W \otimes \mathbb{Q}_{p}$. Choose $n$ such that $a \in p^{-n} V$; then all the $p^{m} z_{m}$ and $p^{m} w_{m}$ lie in $p^{-n} V$, which is compact, so there is an infinite subsequence of $m$ such that the $p^{m} z_{m}$ converge and the $p^{m} w_{m}$ converge. The limits must be equal, since $p^{m} z_{m}-p^{m} w_{m} \in p^{m} V$. The common limit in $(Z \cap W) \otimes \mathbb{Q}_{p}$ represents $a$.

Corollary 5.2. The group $T$ is finite.

Proof. The maximal divisible subgroup of a co-finite-type $\mathbb{Z}_{p}$-module is of finite index.

Corollary 5.3. The exact sequence $0 \rightarrow R \rightarrow S \rightarrow T \rightarrow 0$ splits.

Proof. This follows since $R$ is divisible.

Proposition 5.4. If $q$ is a power of $p$, then $S[q]$ is isomorphic to the intersection $Z / q Z \cap W / q W$ in $V / q V$.

Proof. Intersecting

$$
S=\left(Z \otimes \frac{\mathbb{Q}_{p}}{\mathbb{Z}_{p}}\right) \cap\left(W \otimes \frac{\mathbb{Q}_{p}}{\mathbb{Z}_{p}}\right)
$$

with the $q$-torsion subgroup $\frac{1}{q} V / V$ of $V \otimes \frac{\mathbb{Q}_{p}}{\mathbb{Z}_{p}}$ yields

$$
S[q]=\frac{\frac{1}{q} Z}{Z} \cap \frac{\frac{1}{q} W}{W} .
$$


The multiplication-by- $q$ isomorphism $\frac{1}{q} V / V \rightarrow V / q V$ sends this group to $Z / q Z \cap W / q W$.

\subsection{Model for the Cassels-Tate pairing}

Here we define a natural nondegenerate alternating pairing on $T$. Extend $Q$ to a quadratic form $V \otimes \mathbb{Q}_{p} \rightarrow \mathbb{Q}_{p}$ and define $\langle\rangle:,\left(V \otimes \mathbb{Q}_{p}\right) \times\left(V \otimes \mathbb{Q}_{p}\right) \rightarrow \mathbb{Q}_{p}$ by $\langle x, y\rangle:=Q(x+y)-Q(x)-Q(y)$. Then $\langle,\rangle \bmod \mathbb{Z}_{p}$ identifies $V \otimes \mathbb{Q}_{p}$ with its own Pontryagin dual, and the subgroup

$$
Z^{\perp}:=\left\{v \in V \otimes \mathbb{Q}_{p}:\langle v, z\rangle \bmod \mathbb{Z}_{p}=0 \text { for all } z \in Z\right\}
$$

equals $Z \otimes \mathbb{Q}_{p}+V$. Similarly, $W^{\perp}=W \otimes \mathbb{Q}_{p}+V$.

Suppose that $x, y \in T$. Lift $x$ to $\widetilde{x} \in\left(Z \otimes \frac{\mathbb{Q}_{p}}{\mathbb{Z}_{p}}\right) \cap\left(W \otimes \frac{\mathbb{Q}_{p}}{\mathbb{Z}_{p}}\right)$. Choose $z_{x} \in Z \otimes \mathbb{Q}_{p}$ whose image in $V \otimes \frac{\mathbb{Q}_{p}}{\mathbb{Z}_{p}}$ equals $\widetilde{x}$. Define $w_{x}, \widetilde{y}, z_{y}$, and $w_{y}$ analogously.

Proposition 5.5. The map

$$
\begin{aligned}
{[,]: T \times T } & \rightarrow \frac{\mathbb{Q}_{p}}{\mathbb{Z}_{p}} \\
x, y & \mapsto Q\left(z_{x}-w_{y}\right) \bmod \mathbb{Z}_{p}
\end{aligned}
$$

is well-defined, and it is a nondegenerate alternating bilinear pairing.

Proof. First,

$$
z_{x}-w_{x} \in \operatorname{ker}\left(V \otimes \mathbb{Q}_{p} \rightarrow V \otimes \frac{\mathbb{Q}_{p}}{\mathbb{Z}_{p}}\right)=V
$$

Since $Z$ and $W$ are isotropic,

$$
Q\left(z_{x}-w_{y}\right)=-\left\langle z_{x}, w_{y}\right\rangle=-\left\langle z_{x}-w_{x}, w_{y}\right\rangle,
$$

so changing $w_{y}$ (by an element of $W$ ) changes $Q\left(z_{x}-w_{y}\right)$ by an element of $\langle V, W\rangle \subseteq \mathbb{Z}_{p}$, so $Q\left(z_{x}-w_{y}\right) \bmod \mathbb{Z}_{p}$ is unchanged. Similarly, changing $z_{x}$ (by an element of $Z$ ) does not change $Q\left(z_{x}-w_{y}\right) \bmod \mathbb{Z}_{p}$. If $\widetilde{x}=\widetilde{y}$, then we may choose $w_{y}=w_{x}$, so $Q\left(z_{x}-w_{y}\right)=Q\left(z_{x}-w_{x}\right) \in Q(V) \subseteq \mathbb{Z}_{p}$, so $Q\left(z_{x}-w_{y}\right) \bmod \mathbb{Z}_{p}=0$. Thus we have an alternating bilinear pairing on $\left(Z \otimes \frac{\mathbb{Q}_{p}}{\mathbb{Z}_{p}}\right) \cap\left(W \otimes \frac{\mathbb{Q}_{p}}{\mathbb{Z}_{p}}\right)$ and it remains to show that the kernel on either side is $(Z \cap W) \otimes \frac{\mathbb{Q}_{p}}{\mathbb{Z}_{p}}$ so that it induces a nondegenerate alternating pairing on $T$.

The following are equivalent for $\widetilde{x} \in\left(Z \otimes \frac{\mathbb{Q}_{p}}{\mathbb{Z}_{p}}\right) \cap\left(W \otimes \frac{\mathbb{Q}_{p}}{\mathbb{Z}_{p}}\right)$ : 
- $Q\left(z_{x}-w_{y}\right) \bmod \mathbb{Z}_{p}=0$ for all $\widetilde{y} \in\left(Z \otimes \frac{\mathbb{Q}_{p}}{\mathbb{Z}_{p}}\right) \cap\left(W \otimes \frac{\mathbb{Q}_{p}}{\mathbb{Z}_{p}}\right)$;

- $\left\langle z_{x}-w_{x}, w_{y}\right\rangle \in \mathbb{Z}_{p}$ for all $\tilde{y} \in\left(Z \otimes \frac{\mathbb{Q}_{p}}{\mathbb{Z}_{p}}\right) \cap\left(W \otimes \frac{\mathbb{Q}_{p}}{\mathbb{Z}_{p}}\right)$;

- $\left\langle z_{x}-w_{x}, w\right\rangle \in \mathbb{Z}_{p}$ for all $w \in\left(Z \otimes \mathbb{Q}_{p}+V\right) \cap\left(W \otimes \mathbb{Q}_{p}+V\right)=Z^{\perp} \cap W^{\perp}$;

- $z_{x}-w_{x} \in\left(Z^{\perp} \cap W^{\perp}\right)^{\perp}=Z+W$; and

- $\widetilde{x} \in(Z \cap W) \otimes \frac{\mathbb{Q}_{p}}{\mathbb{Z}_{p}}$.

\subsection{Predictions for rank}

Corollary 2.2 defines a probability measure on $\mathrm{OGr}_{n}\left(\mathbb{Z}_{p}\right)$. In Section 1.2 when describing the process leading to $\mathscr{Q}_{2 n}$ (which will be proved to be well-defined in Section 5.4), we chose both $Z$ and $W$ randomly from $\operatorname{OGr}_{n}\left(\mathbb{Z}_{p}\right)$. But since the orthogonal group of $(V, Q)$ acts transitively on $\mathrm{OGr}_{V}\left(\mathbb{Z}_{p}\right)$, fixing $W$ to be $\mathbb{Z}_{p}^{n} \times 0$ and choosing only $Z$ at random would produce the same distribution. A similar comment applies to $\mathscr{T}_{2 n, r}$. From now on, we assume that $W$ is fixed as above.

Proposition 5.6. Fix $n$. If $Z$ is chosen randomly from $\mathrm{OGr}_{n}\left(\mathbb{Z}_{p}\right)$, then the $\mathbb{Z}_{p}$-module $Z \cap W$ is free of rank 0 with probability $1 / 2$, and free of rank 1 with probability $1 / 2$.

Proof. By Proposition 4.9, $\operatorname{dim} \mathcal{S}_{n, r}<\operatorname{dim} \mathrm{OGr}_{n}$ for $r \geq 2$, so the probability that $\operatorname{rk}(Z \cap W) \geq 2$ is 0 by Proposition 2.1(b). On the other hand, $\mathrm{OGr}_{n}^{\text {even }}$ and $\mathrm{OGr}_{n}^{\text {odd }}$ are isomorphic by Proposition $4.5(\mathrm{a})$, so the parity of $\operatorname{rk}(Z \cap W)$ is equidistributed.

Conjecture 1.3 implies that the distribution of $E(k) \otimes \frac{\mathbb{Q}_{p}}{\mathbb{Z}_{p}}$ matches that of $(Z \cap W) \otimes \frac{\mathbb{Q}_{p}}{\mathbb{Z}_{p}}$, or equivalently that the distribution of $E(k) \otimes \mathbb{Z}_{p}$ matches that of $Z \cap W$. Thus it implies that $50 \%$ of elliptic curves over $k$ have rank 0 , and $50 \%$ have rank 1 .

\subsection{Random models and their compatibility}

One can show that the locus of $Z \in \mathrm{OGr}_{n}\left(\mathbb{Z}_{p}\right)$ for which the sequence $0 \rightarrow$ $R \rightarrow S \rightarrow T \rightarrow 0$ is isomorphic to a given sequence is locally closed in the $p$-adic topology, and hence measurable. This would show that $\mathscr{Q}_{2 n}$ is welldefined. A similar argument using the probability measure on $\mathcal{S}_{n, r}\left(\mathbb{Z}_{p}\right)$ would show that $\mathscr{T}_{2 n, r}$ is well-defined. We find it more convenient, however, to prove these measurability claims and to prove that $\lim _{n \rightarrow \infty} \mathscr{Q}_{2 n}$ and $\lim _{n \rightarrow \infty} \mathscr{T}_{2 n, r}$ exist by relating them to the distributions $\mathscr{A}_{n, r}$. Recall that we already proved in Section 3.8 that the $\mathscr{A}_{n, r}$ exist and converge to a limit $\mathscr{A}_{r}$ as $n \rightarrow \infty$ through integers with $n-r \in 2 \mathbb{Z}_{\geq 0}$. 
Before giving the proof that the $\operatorname{limit}_{\lim _{n \rightarrow \infty}} \mathscr{T}_{2 n, r}=: \mathscr{T}_{r}$ exists and coincides with $\mathscr{A}_{r}$ (Theorem 1.10(b)), let us explain the idea. There is a simple relationship between alternating matrices $A$ and maximal isotropic direct summands $Z$ : namely, if we view $A$ as a linear map $W \rightarrow W^{T}$, then $Z:=\operatorname{graph}(A) \subset W \oplus W^{T}$ is maximal isotropic. But not every maximal isotropic direct summand $Z \leq W \oplus W^{T}$ comes from an $A$. Over a field, the $Z$ 's that arise are those that intersect $W^{T}$ trivially; at the other extreme is $W^{T}$ itself; a general $Z$ is a hybrid of these two extremes: namely, such a $Z$ arises by writing $W=W_{1} \oplus W_{2}$, forming the corresponding decomposition $W^{T}=W_{1}^{T} \oplus W_{2}^{T}$, and taking $Z:=W_{1}^{T} \oplus \operatorname{graph}(A)$ for some alternating $A: W_{2} \rightarrow W_{2}^{T}$. We need to work over $\mathbb{Z}_{p}$ instead of a field, but we can still represent a general $Z$ in terms of a decomposition as above (note, however, that the $Z$ 's that arise directly from an $A$ on the whole of $W$ are those for which the $\bmod p$ reductions of $Z$ and $W^{T}$ intersect trivially). Moreover, we will show that the uniform distribution of $Z \in \mathcal{S}_{n, r}$ can be obtained by choosing the decompositions of $W$ and $W^{T}$ at random (with respect to a suitable measure) and then choosing $A: W_{2} \rightarrow W_{2}^{T}$ at random from those alternating maps whose kernel has rank $r$. The distribution $\mathscr{T}_{2 n, r}$ is defined in terms of the group $T$ arising from $Z$. It turns out that $T \simeq(\text { coker } A)_{\text {tors }}$, and one shows that with high probability as $n \rightarrow \infty$, the size of $A$ is large, so the distribution of $(\operatorname{coker} A)_{\text {tors }}$ is well approximated by $\mathscr{A}_{r}$.

Proofs of Theorems 1.6 and 1.10(b). We use the notation $\bar{V}:=V / p V$. Fix a maximal isotropic $\mathbb{F}_{p}$-subspace $\Lambda \leq \bar{V}$ with respect to the $\mathbb{F}_{p}$-valued quadratic form $(Q \bmod p)$ such that $\Lambda \cap \bar{W}=0$ in $\bar{V}$. Define a distribution $\mathscr{G}$ on submodules $Z \leq V$ as follows:

1. Choose a maximal isotropic direct summand $W^{T} \leq V$ at random conditioned on $\overline{W^{T}}=\Lambda$. (Then $\left.\langle\rangle\right|_{,W \times W^{T}}$ is nondegenerate $\bmod p$, so it identifies $W^{T}$ with the $\mathbb{Z}_{p}$-dual of $W$, so the name $W^{T}$ makes sense. Also, $V=W \oplus W^{T}$.)

2. Choose $m \in\{0,1, \ldots, n-r\}$ at random so that its distribution matches the distribution of $\operatorname{dim}(\overline{\mathcal{Z}} \cap \Lambda)$ for $\mathcal{Z}$ chosen from $\mathcal{S}_{n, r}\left(\mathbb{Z}_{p}\right)$. The scheme $\mathcal{S}_{n, r}$ is contained in $\operatorname{OGr}_{n}^{\text {parity }(r)}$, so $\operatorname{dim}(\overline{\mathcal{Z}} \cap \bar{W}) \equiv r(\bmod 2)$. Corollary 4.7 applied to $(\mathcal{Z}, \bar{W}, \Lambda)$ implies that $m+r \equiv n(\bmod 2)$.

3. Choose a random $\mathbb{Z}_{p}$-module decomposition of $W$ as $W_{1} \oplus W_{2}$ such that rk $W_{1}=m$. Let $W^{T}=W_{1}^{T} \oplus W_{2}^{T}$ be the induced decomposition of $W^{T}$; i.e., $W_{2}^{T}$ is the annihilator of $W_{1}$ with respect to $\left.\langle\rangle\right|_{,W \times W^{T}}$, and $W_{1}^{T}$ is the annihilator of $W_{2}$. (Then $W_{i}^{T}$ is isomorphic to the $\mathbb{Z}_{p}$-dual of $W_{i}$ for $i=1,2$.) 
4. Choose an alternating $\mathbb{Z}_{p}$-linear map $A: W_{2} \rightarrow W_{2}^{T}$ at random from maps whose kernel has rank $r$ (since $\operatorname{rk} W_{2}=n-m \equiv r(\bmod 2)$, the set of such $A$ is nonempty). Let $\operatorname{graph}(A) \leq W_{2} \times W_{2}^{T}$ be its graph. Let $Z=W_{1}^{T} \oplus \operatorname{graph}(A)$.

Since $A$ is alternating, the direct summand graph $(A)$ of $V$ is isotropic. Since $W_{1}^{T} \leq W^{T}$, the direct summand $W_{1}^{T}$ is isotropic. Under $\left.\langle\rangle\right|_{,W \times W^{T}}$, the direct summand $W_{1}^{T}$ annihilates $W_{2}^{T}$ (since both are contained in $W^{T}$ ) and $W_{2}$ (by definition). The previous three sentences show that $Z$ is an isotropic direct summand. Its rank is $\operatorname{rk} W_{1}^{T}+\operatorname{rk} W_{2}=\operatorname{rk} W_{1}+\operatorname{rk} W_{2}=n$, so $Z$ is a maximal isotropic direct summand.

Reducing modulo $p$ yields

$$
\bar{Z}=\overline{W_{1}^{T}} \oplus \operatorname{graph}(\bar{A}),
$$

so in $\bar{V}$ we have

$$
\bar{Z} \cap \Lambda=\bar{Z} \cap \overline{W^{T}}=\overline{W_{1}^{T}},
$$

which is of $\mathbb{F}_{p}$-dimension $m$.

Claim: $\mathscr{G}$ coincides with the uniform distribution on $\mathcal{S}_{n, r}\left(\mathbb{Z}_{p}\right)$. In the construction of $\mathscr{G}$, if we condition on $\bar{W}_{1}$ and $\bar{W}_{2}$, then $W_{1}$ and $W_{2}$ are uniformly distributed among the $\mathbb{Z}_{p}$-submodules of $W$ reducing to $\bar{W}_{1}$ and $\bar{W}_{2}$. Also, $W^{T}$ is uniform among maximal isotropic direct summands of $V$ reducing to $\Lambda$, so $W_{1}^{T}$ is uniform among rank $m$ isotropic direct summands of $V$ with $\overline{W_{1}^{T}} \subseteq \Lambda$. Similarly, if we condition on $\operatorname{graph}(\bar{A})$, then $\operatorname{graph}(A)$ is uniformly distributed among the isotropic $\mathbb{Z}_{p}$-modules reducing to graph $(\bar{A})$. Thus if we condition on $\bar{Z}$, then $\mathscr{G}$ assigns the uniform measure to the set of maximal isotropic direct summands $Z$ lifting $\bar{Z}$ such that $\operatorname{dim}(Z \cap W)=r$.

The uniform distribution on $\mathcal{S}_{n, r}\left(\mathbb{Z}_{p}\right)$ has the same property, so to prove the claim, it suffices to show that the distributions of $\bar{Z}$ match. For each $m$, both distributions for $\bar{Z}$ are uniform over all maximal isotropic subspaces of $\bar{V}$ for which $\operatorname{dim}(\bar{Z} \cap \bar{W}) \geq r$ and $\operatorname{dim}(\bar{Z} \cap \Lambda)=m$, so it suffices to prove that the distribution of the integer $\operatorname{dim}(\bar{Z} \cap \Lambda)$ is the same for both distributions. The latter holds by the choice of $m$. This proves the claim.

For $Z$ sampled from $\mathscr{G}$, the definition of $Z$ yields

$$
\begin{aligned}
\left(Z \otimes \frac{\mathbb{Q}_{p}}{\mathbb{Z}_{p}}\right) \cap\left(W \otimes \frac{\mathbb{Q}_{p}}{\mathbb{Z}_{p}}\right) & =\operatorname{graph}\left(A \otimes \frac{\mathbb{Q}_{p}}{\mathbb{Z}_{p}}\right) \cap\left(W \otimes \frac{\mathbb{Q}_{p}}{\mathbb{Z}_{p}}\right) \\
& \simeq \operatorname{ker}\left(A \otimes \frac{\mathbb{Q}_{p}}{\mathbb{Z}_{p}}\right),
\end{aligned}
$$


whose Pontryagin dual is coker $A$. The quotient $T$ of the left side by its maximal divisible subgroup $(Z \cap W) \otimes \frac{\mathbb{Q}_{p}}{\mathbb{Z}_{p}}$ is dual to the finite group (coker $\left.A\right)_{\text {tors }}$, hence isomorphic to (coker $A)_{\text {tors }}$. Thus the distribution $\mathscr{T}_{2 n, r}$ of $T$ is a weighted average over $m$ of the distribution $\mathscr{A}_{n-m, r}$ of $(\operatorname{coker} A)_{\text {tors }}$ for $A \in \mathcal{A}_{n-m, r}$; this proves in particular that $\mathscr{T}_{2 n, r}$ is well-defined.

We next show that as $n \rightarrow \infty$, the probability that $m$ is small, say less than $n / 2$, tends to 1 . In fact, we show that this holds even after conditioning on the intersection $\overline{\mathcal{Z}} \cap \bar{W}$; i.e., we will prove that

$$
\inf _{Y} \operatorname{Prob}(m<n / 2 \mid \overline{\mathcal{Z}} \cap \bar{W}=Y) \rightarrow 1
$$

as $n \rightarrow \infty$, where $Y$ ranges over the possibilities for $\overline{\mathcal{Z}} \cap \bar{W}$. Fix $Y$. Let $y:=\operatorname{dim} Y$. Since $m+y \leq \operatorname{dim} \overline{\mathcal{Z}}=n$, the probability is 1 if $y>n / 2$, so assume that $y \leq n / 2$. The subgroup of $\mathrm{O}_{2 n}\left(\mathbb{Z}_{p}\right)$ preserving $W$ and $Y$ acts transitively on the maximal isotropic subspaces $\overline{\mathcal{Z}}$ of $\mathbb{F}_{p}^{2 n}$ satisfying $\overline{\mathcal{Z}} \cap \bar{W}=Y$, by Lemma 4.3, so the distribution of $\overline{\mathcal{Z}}$ is uniform among such subspaces. Thus $\overline{\mathcal{Z}} / Y$ is a uniformly random maximal isotropic subspace of $Y^{\perp} / Y$ intersecting $\bar{W} / Y$ trivially. In $Y^{\perp} / Y$, the image of $\Lambda \cap Y^{\perp}$ is a maximal isotropic complement $C$ of $\bar{W} / Y$, so $\overline{\mathcal{Z}} / Y$ is the graph of a uniformly random alternating map $B: C \rightarrow \bar{W} / Y$ (see Lemma 4.1(b)). Then $m=\operatorname{dim} \operatorname{ker} B$. By Lemma $3.7, m<(n-y) / 2$ with high probability, so $m<n / 2$ with high probability.

So the size $n-m$ of the matrix $A$ is large with high probability, and we have already seen that $n-m \equiv r(\bmod 2)$. Thus the weighted average converges as $n \rightarrow \infty$ to $\mathscr{A}_{r}$. In other words, $\mathscr{T}_{r}$ exists and coincides with $\mathscr{A}_{r}$.

We now prove that the distributions $\mathscr{Q}_{2 n}$ exist and converge to $\mathscr{Q}$ as $n \rightarrow \infty$.

Proof of Theorem 1.2. Define a new distribution $\mathscr{Q}_{2 n}^{\prime}$ on isomorphism classes of short exact sequences as follows. Choose $r \in\{0,1\}$ uniformly at random, and let $R=\left(\mathbb{Q}_{p} / \mathbb{Z}_{p}\right)^{r}$. Choose $T$ with respect to the distribution $\mathscr{T}_{2 n, r}$. Form the exact sequence

$$
0 \longrightarrow R \longrightarrow R \oplus T \longrightarrow T \rightarrow 0
$$

By Proposition 5.6 and Corollary 5.3, the distribution $\mathscr{Q}_{2 n}$ coincides with $\mathscr{Q}_{2 n}^{\prime}$; in particular, it is well-defined.

For each $r$, the distribution $\mathscr{T}_{2 n, r}$ tends to a limit as $n \rightarrow \infty$, so the same is true of $\mathscr{Q}_{2 n}^{\prime}=\mathscr{Q}_{2 n}$. 


\subsection{Predictions for $\operatorname{Sel}_{p^{e}}$}

Lemma 5.7. Fix a global field $k$. Asymptotically $100 \%$ of elliptic curves over $k$ satisfy $E(k)_{\text {tors }}=0$.

Proof. For each global field $k$ and prime $p$, the theory of modular curves and Igusa curves shows that the generic elliptic curve (over $k\left(a_{1}, a_{2}, a_{3}, a_{4}, a_{6}\right)$ ) has no nonzero rational $p$-torsion point. By the Hilbert irreducibility theorem, the same holds for asymptotically $100 \%$ of elliptic curves over $k$. The size of the torsion subgroup is bounded by a constant depending only on $k$ [Lev68, Maz77, KM95, Mer96], so we need consider only finitely many $p$. Thus $100 \%$ of $E \in \mathscr{E}$ satisfy $E(k)_{\text {tors }}=0$.

Remark 5.8. One could also prove Lemma 5.7 without using [Mer96]: the torsion subgroup can also be controlled by using reduction modulo primes.

Proposition 5.9. Suppose that $E$ is an elliptic curve over a global field $k$ with $E(k)_{\text {tors }}=0$. Let $m$ and $n$ be positive integers such that char $k \nmid m, n$ and $m \mid n$. Then

(a) The inclusion $E[m] \rightarrow E[n]$ induces an isomorphism

$$
\mathrm{H}^{1}(k, E[m]) \rightarrow \mathrm{H}^{1}(k, E[n])[m]
$$

(b) This isomorphism identifies $\operatorname{Sel}_{m} E$ with $\left(\operatorname{Sel}_{n} E\right)[m]$.

(c) If $p$ is a prime number and $e \in \mathbb{Z}_{\geq 0}$, then $\operatorname{Sel}_{p^{e}} E \simeq\left(\operatorname{Sel}_{p^{\infty}} E\right)\left[p^{e}\right]$.

Proof.

(a) Taking cohomology of $0 \rightarrow E[m] \rightarrow E[n] \stackrel{m}{\rightarrow} E[n / m] \rightarrow 0$ yields a homomorphism $\alpha$ fitting into the exact sequence

$$
0 \rightarrow \mathrm{H}^{1}(k, E[m]) \rightarrow \mathrm{H}^{1}(k, E[n]) \stackrel{\alpha}{\rightarrow} \mathrm{H}^{1}(k, E[n / m]) .
$$

Replacing $m$ by $n / m$ shows that $E[n / m] \hookrightarrow E[n]$ induces an injection $\mathrm{H}^{1}(k, E[n / m]) \rightarrow \mathrm{H}^{1}(k, E[n])$. The composition $E[n] \stackrel{m}{\rightarrow} E[n / m] \hookrightarrow E[n]$ induces a composition $\mathrm{H}^{1}(k, E[n]) \stackrel{\alpha}{\rightarrow} \mathrm{H}^{1}(k, E[n / m]) \hookrightarrow \mathrm{H}^{1}(k, E[n])$ that equals multiplication by $m$, so $\mathrm{H}^{1}(k, E[m]) \simeq \operatorname{ker} \alpha \simeq \mathrm{H}^{1}(k, E[n])[m]$.

(b) An element of $\mathrm{H}^{1}(k, E[m])$ lies in the subgroup $\operatorname{Sel}_{m} E$ if and only if its image in $\mathrm{H}^{1}(k, E[n])$ lies in $\operatorname{Sel}_{n} E$, since the condition for either is that it map to 0 in $\mathrm{H}^{1}\left(k_{v}, E\right)$ for every $v$.

(c) Apply (b) to $p^{e} \mid p^{n}$ and take the direct limit as $n \rightarrow \infty$. 
Let $q=p^{e}$ for some prime $p$ and $e \geq 0$. Because of Propositions 5.4 and 5.9(c), Conjecture 1.3 implies that the distribution of $\operatorname{Sel}_{q} E$ is the limit as $n \rightarrow \infty$ of the distribution of $Z \cap W$ for random $Z, W \in \mathrm{OGr}_{n}(\mathbb{Z} / q \mathbb{Z})$. Taking $q=p$, we recover [PR12, Conjecture 1.1(a)].

Given $m \in \mathbb{Z}_{\geq 0}$ and a finite $\mathbb{Z} / q \mathbb{Z}$-module $G$, let $I_{m}(G)$ be the number of injective homomorphisms $(\mathbb{Z} / q \mathbb{Z})^{m} \rightarrow G$. An inclusion-exclusion argument shows that $I_{m}(G)$ is a monic degree $m$ polynomial in \# $G$ with coefficients in $\mathbb{Z}[q]$. Thus if $G$ is sampled from some distribution on finite $\mathbb{Z} / q \mathbb{Z}$-modules, then knowledge of the averages of $I_{m}(G)$ for all $m \geq 0$ is equivalent to knowledge of all moments of $\# G$. For the distribution of $Z \cap W$ for $Z, W \in$ $\mathrm{OGr}_{n}(\mathbb{Z} / q \mathbb{Z})$, it turns out that the formulas for the averages of $I_{m}(G)$ are simpler than the formulas for the moments:

Theorem 5.10. Fix $m \in \mathbb{Z}_{\geq 0}$. The average of $I_{m}(Z \cap W)$ as $Z, W$ vary over $\mathrm{OGr}_{n}(\mathbb{Z} / q \mathbb{Z})$ tends to $q^{m(m+1) / 2}$ as $n \rightarrow \infty$.

Proof. For each $n$, we may fix $W$. The desired number is the number of injective homomorphisms $h:(\mathbb{Z} / q \mathbb{Z})^{m} \rightarrow W$ times the probability that a random $Z \in \mathrm{OGr}_{n}(\mathbb{Z} / q \mathbb{Z})$ contains $\operatorname{im}(h)$. The number of $h$ 's is

$$
I_{m}(W)=(\# W)^{m} \prod_{i=0}^{m-1}\left(1-p^{i-n}\right) .
$$

The $Z$ 's containing $\operatorname{im}(h)$ correspond to the maximal isotropic direct summands of $\operatorname{im}(h)^{\perp} / \operatorname{im}(h)$, a hyperbolic quadratic $\mathbb{Z} / q \mathbb{Z}$-module of rank $2 n-$ $2 m$, so their number is $\# \mathrm{OGr}_{n-m}(\mathbb{Z} / q \mathbb{Z})$. Using Lemma 4.8, we compute

(9) $I_{m}(W) \frac{\# \mathrm{OGr}_{n-m}(\mathbb{Z} / q \mathbb{Z})}{\# \mathrm{OGr}_{n}(\mathbb{Z} / q \mathbb{Z})}=q^{m(m+1) / 2} \prod_{i=0}^{m-1}\left(1-p^{i-n}\right) \prod_{i=n-m}^{n-1}\left(1+p^{-i}\right)$,

which tends to $q^{m(m+1) / 2}$ as $n \rightarrow \infty$.

Remark 5.11. The average in Theorem 5.10 is unchanged if we condition on the event that $\operatorname{dim}(Z \cap W)$ has a prescribed parity, because this amounts to replacing each of $\mathrm{OGr}_{n}$ and $\mathrm{OGr}_{n-m}$ in (9) by one of its two components, once $n>m$.

Theorem 5.10 suggests the following:

Conjecture 5.12. For each $m \geq 0$, the average of $I_{m}\left(\operatorname{Sel}_{q} E\right)$ over $E \in \mathscr{E}$ exists and equals $q^{m(m+1) / 2}$. 
Remark 5.13. The combination of Conjecture 1.3 and Theorem 5.10 does not quite imply Conjecture 5.12 , because it could be that a density 0 subset of $\mathscr{E}$ contributes a positive amount towards the average. But if we assume also the weak conjecture that every moment of \# $\operatorname{Sel}_{q} E$ is bounded (in the limsup sense), then the boundedness of the $(m+1)^{\text {st }}$ moment implies that no density 0 subset of $\mathscr{E}$ contributes a positive amount towards the $m^{\text {th }}$ moment, so the average of $I_{m}\left(\operatorname{Sel}_{q} E\right)$ for $E \in \mathscr{E}$ is $q^{m(m+1) / 2}$.

Remark 5.14. The case of Theorem 5.10 in which $q$ is a prime $p$ is equivalent to [PR12, Proposition 2.22(a)], which states that the $m^{\text {th }}$ moment of $\#(Z \cap$ $W$ ) equals $\prod_{i=1}^{m}\left(p^{i}+1\right)$. With more work, one could calculate the moments also for non-prime $q$, but the answers appear to be complicated. See [DJ14a] for an analogous calculation of the conjectural moments of $\# \amalg\left[p^{e}\right]$.

Remark 5.15. For small values of $q$ and $m=1$, the result of Theorem 5.10 can be related to the Tamagawa number $\tau\left(\mathrm{PGL}_{q}\right)=q$. (See [BS15a, BS15b] and [Poo13].) Is there a Tamagawa number explanation for all $q$ and $m$ ?

\subsection{Considering all $p$-primary parts at once}

Let $\operatorname{Sel} E:=\lim _{\longrightarrow} \operatorname{Sel}_{n} E$ be the direct limit over all $n \in \mathbb{Z}_{>0}$, ordered by divisibility, so $\overrightarrow{\mathrm{Sel}} E \simeq \bigoplus_{p} \operatorname{Sel}_{p^{\infty}} E$. It fits in an exact sequence

$$
0 \longrightarrow E(k) \otimes \frac{\mathbb{Q}}{\mathbb{Z}} \longrightarrow \operatorname{Sel} E \longrightarrow \amalg \longrightarrow 0
$$

of discrete $\widehat{\mathbb{Z}}$-modules (i.e., torsion abelian groups). The $p$-primary parts of this sequence should not be completely independent, because if $\amalg$ is finite, then the $\mathbb{Z}_{p}$-corank of the $p$-primary part $\operatorname{Sel}_{p^{\infty}} E$ of $\operatorname{Sel} E$ is independent of $p$.

Therefore we condition on the rank $r$, in which case we need only focus on the model for $\amalg$. Here is our model: independently for each prime $p$, choose a finite symplectic abelian $p$-group $T_{p}$ with respect to $\mathscr{T}_{r}$ (or equivalently $\mathscr{A}_{r}$, by Theorem $1.10(\mathrm{~b})$ ), and define $T:=\bigoplus_{p} T_{p}$.

Theorem 5.16. If $r \geq 1$, then the group $T$ above is finite with probability 1 , and has the distribution of [Del01, Heuristic Assumption], with the correction that $r / 2$ is replaced by $r$.

Proof. By Theorem 3.11 for $G=0$,

$$
\operatorname{Prob}\left(T_{p} \neq 0\right)=1-\prod_{i=r+1}^{\infty}\left(1-p^{1-2 i}\right)=O\left(p^{-1-2 r}\right)
$$


If $r \geq 1$, then $\sum_{p} \operatorname{Prob}\left(T_{p} \neq 0\right)$ converges, so the Borel-Cantelli lemma implies that $T_{p}=0$ for all but finitely $p$ with probability 1 , so $T$ is finite with probability 1 . The probability that $T$ is isomorphic to a given symplectic abelian group $G$ is the (convergent) product over $p$ of the probability that $T_{p} \simeq G\left[p^{\infty}\right]$. Since the formula in [Del01, Heuristic Assumption] is multiplicative on $p$-primary parts, the result follows.

For the rest of this section, assume that $r=0$. Then $\sum_{p} \operatorname{Prob}\left(T_{p} \neq 0\right)$ diverges, and the probability that $T$ is isomorphic to any particular finite abelian group is 0 , so we do not obtain a discrete probability distribution on finite abelian groups. This situation is similar to that for class groups of imaginary quadratic fields: the density of such fields having a specified class group is 0 . In the class group setting, the article [CL84] formulated nontrivial statements by measuring the probability not of individual groups but of certain infinite sets of isomorphism classes of groups, and more generally, by computing the average of certain functions $f$ defined on such isomorphism classes. Following [Del01], we will do something analogous for symplectic abelian groups.

Let $\mathscr{E}_{0,<X}$ be the set of $E \in \mathscr{E}_{0}$ of height less than $X$. We use $\sum_{G}$ to denote a sum over (isomorphism classes of) symplectic abelian groups; we often restrict the sum by imposing conditions on the size of $G$. For a symplectic abelian group $G$, define

$$
w_{G}:=\frac{\# G}{\# \operatorname{Sp}(G)} .
$$

For $k=\mathbb{Q}$, Delaunay [Del01, Heuristic Assumption], inspired by [CL84], proposed the heuristic

$$
\lim _{X \rightarrow \infty} \frac{\sum_{E \in \mathscr{E}_{0,<X}} f(\amalg(E))}{\sum_{E \in \mathscr{E}_{0,<X}} 1} \stackrel{?}{=} \lim _{n \rightarrow \infty} \frac{\sum_{\# G \leq n} f(G) w_{G}}{\sum_{\# G \leq n} w_{G}} .
$$

Some hypotheses on $f$ are necessary since one can construct wildly oscillating functions $f$ for which even the "easy" limit on the right side of (10) fails to exist. Let us now describe a class of functions for which we expect equality in (10). Fix a set of primes $P$ such that $\sum_{p \in P} 1 / p<\infty$. Given $G$, write $G=H_{G} \times H_{G}^{\prime}$ where $\# H_{G}$ is divisible only by primes in $P$, and $\# H_{G}^{\prime}$ is divisible only by primes not in $P$. We use $\sum_{H}$ (resp. $\sum_{H^{\prime}}$ ) to denote a sum restricted to symplectic abelian groups of order divisible only by primes in $P$ (resp., not in $P$ ); again we may also impose restrictions on the size of $H$ or $H^{\prime}$. Then $\sum_{p \in P} \operatorname{Prob}\left(T_{p} \neq 0\right) \leq \sum_{p \in P} O(1 / p)<\infty$, so 
the Borel-Cantelli lemma implies that the random group $\bigoplus_{p \in P} T_{p}$ is given by a discrete probability distribution on the set of isomorphism classes of (finite) symplectic abelian groups $H$ of order divisible only by primes in $P$; in fact, Theorem 3.9 implies that $\operatorname{Prob}\left(\bigoplus_{p \in P} T_{p} \simeq H\right)=c_{P} w_{H}$, where $c_{P}$ is a normalizing constant defined as the convergent product $\prod_{p \in P} \prod_{i=1}^{\infty}\left(1-p^{1-2 i}\right)$; in particular, $\sum_{H} w_{H}<\infty$. By an $L^{1}$ function on the set of such $H$, we mean a real-valued function $f$ such that $\sum_{H}|f(H)| w_{H}<\infty$; in particular, bounded functions are $L^{1}$. Given such an $L^{1}$ function $f$, we define

$$
\int f:=\frac{\sum_{H} f(H) w_{H}}{\sum_{H} w_{H}}
$$

and extend $f$ to all symplectic abelian groups $G$ by defining $f(G):=f\left(H_{G}\right)$. It is reasonable to conjecture (10) for $L^{1}$ functions $f$. On the other hand, our model suggests the conjecture that

$$
\lim _{X \rightarrow \infty} \frac{\sum_{E \in \mathscr{E}_{0,<X}} f(\amalg(E))}{\sum_{E \in \mathscr{E}_{0,<X}} 1} \stackrel{?}{=} \int f
$$

for such $L^{1}$ functions $f$. We now prove that Delaunay's prediction agrees with ours, i.e., that the right sides of (10) and (11) are equal.

Theorem 5.17. Let $P$ be a set of primes such that $\sum_{p \in P} 1 / p<\infty$. Let $f$ be an $L^{1}$ function on the set of (isomorphism classes of) symplectic abelian groups $H$ of order divisible only by primes in $P$. Extend $f$ to all symplectic abelian groups $G$ by defining $f(G):=f\left(H_{G}\right)$. Then

$$
\lim _{n \rightarrow \infty} \frac{\sum_{\# G \leq n} f(G) w_{G}}{\sum_{\# G \leq n} w_{G}}=\int f
$$

Before starting the proof of Theorem 5.17, we prove bounds on sums involving $w_{G}$.

Lemma 5.18. For any $N \geq 1$, we have $1 / N \leq \sum_{\# G=N^{2}} w_{G} \leq 2 / N$.

Proof. The sum in Theorem 3.11 being 1 implies that

$$
\sum_{k=0}^{\infty} \sum_{\# G=p^{2 k}} w_{G} t^{k}=\prod_{i=1}^{\infty}\left(1-p^{1-2 i} t\right)^{-1}
$$

holds for $t=p^{-2 r}$ for all $r \in \mathbb{Z}_{\geq 0}$, so it holds identically in $\mathbb{Q}[[t]]$. Apply the $q$-binomial theorem to the right side (take $x=p^{-2}$ and $z=p t$ in the 
expression $Z$ in [Eul48, §313]) and equate coefficients of $t^{k}$ to obtain

$$
\sum_{\# G=p^{2 k}} w_{G}=p^{-k} \prod_{j=1}^{k}\left(1-p^{-2 j}\right)^{-1}
$$

(which is equivalent to [Del01, Corollary 6]). Take the product over the prime powers in the factorization of a positive integer $N$, and use

$$
1 \leq \prod_{\text {prime powers }}\left(1-m^{-2}\right)^{-1} \leq \prod_{m=2}^{\infty}\left(1-m^{-2}\right)^{-1}=2 .
$$

Corollary 5.19. We have

$$
\sum_{\# G \leq n} w_{G} \geq \frac{1}{2} \log n \quad \text { and } \quad \sum_{\# G \in[\ell, n]} w_{G}=O(\log (n / \ell)) .
$$

Proof of Theorem 5.17. We may add a constant to $f$ in order to assume that $\int f=0$; in other words, $\sum_{H} f(H) w_{H}=0$. For any $M \in \mathbb{R}$, define $S_{M}:=\sum_{\# H \leq M} f(H) w_{H}$; thus the $S_{M}$ are bounded and $\lim _{M \rightarrow \infty} S_{M}=0$. Suppose that $\epsilon>0$ is given; fix $m$ such that $M>m$ implies $\left|S_{M}\right|<\epsilon$. Then

$$
\begin{aligned}
\left|\sum_{\# G \leq n} f(G) w_{G}\right| & =\left|\sum_{\# H^{\prime} \leq n} \sum_{\# H \leq \frac{n}{\# H^{\prime}}} f(H) w_{H} w_{H^{\prime}}\right| \\
& \leq \sum_{\# H^{\prime} \leq n} w_{H^{\prime}}\left|S_{n / \# H^{\prime}}\right| \\
\leq & \sum_{\# H^{\prime}<n / m} w_{H^{\prime}} \epsilon+\sum_{\# H^{\prime} \in[n / m, n]} w_{H^{\prime}} O(1) \\
\leq & \left(\frac{1}{2} \log n\right) \epsilon+O(\log m) \quad \text { (by Corollary 5.19) }
\end{aligned}
$$

and

$$
\sum_{\# G \leq n} w_{G} \geq \frac{1}{2} \log n \quad \text { (by Corollary 5.19). }
$$

Thus the lim sup of the absolute value of the ratio in (12) is bounded by $\epsilon$. This holds for every $\epsilon$, so the limit is 0 , matching $\int f$. 


\subsection{Prediction for the average size of the $n$-Selmer group}

The model in Section 5.6 suggests a distribution for $\mathrm{Sel}_{n} E$. Namely, choose $r \in\{0,1\}$ uniformly at random, construct $T=\bigoplus T_{p}$ as in the sentence before Theorem 5.16, and define $S_{n}:=(\mathbb{Z} / n \mathbb{Z})^{r} \oplus T[n]$.

Proposition 5.20. The average size of $S_{n}$ is $\sigma(n)$.

Proof. In fact, this holds even if we condition on $r$. Then the $p$-primary parts of $S_{n}$ for different $p$ are independent, so we may assume that $n$ is a prime power, say $p^{e}$. Grouping elements by their order shows that

$$
\# S_{p^{e}}=\sum_{f=0}^{e} I_{1}\left(S_{p^{e}}\left[p^{f}\right]\right)
$$

and $S_{p^{e}}\left[p^{f}\right]$ has the same distribution as $S_{p^{f}}$, so the result follows from Theorem 5.10 and Remark 5.11.

If we assume that for each finite abelian group $G$ the density of $E \in \mathscr{E}$ satisfying $\operatorname{Sel}_{n} E \simeq G$ equals the probability that $S_{n} \simeq G$, and also that the average of $\left(\# \operatorname{Sel}_{n} E\right)^{2}$ in the lim sup sense is finite (so that density 0 subsets of $\mathscr{E}$ do not contribute to the average of \# $\operatorname{Sel}_{n} E$ ), then the average of $\# \operatorname{Sel}_{n} E$ equals the average of $\# S_{n}$, which is $\sigma(n)$. For $n \leq 5$, this conclusion has been proved [BS15a, BS15b, BS13a, BS13b].

\section{Arithmetic justification}

In this section, we prove results on the arithmetic of elliptic curves that partially explain why $\operatorname{Sel}_{p^{e}} E$ should behave like an intersection of maximal isotropic direct summands.

\subsection{Shafarevich-Tate groups of finite group schemes}

For any $G_{k}$-module or finite $k$-group scheme $M$, define

$$
\amalg^{1}(k, M):=\operatorname{ker}\left(\mathrm{H}^{1}(k, M) \rightarrow \prod_{v \in \Omega} \mathrm{H}^{1}\left(k_{v}, M\right)\right) .
$$

(If $M$ is not étale, then the cohomology should be interpreted as fppf cohomology.) As in [PR12, Theorem 4.14(b)], we will need $\amalg^{1}\left(k, E\left[p^{e}\right]\right)=0$ to hold often so that it is $\operatorname{Sel}_{p^{e}} E$, and not just its image in the group $\mathrm{H}^{1}\left(\mathbf{A}, E\left[p^{e}\right]\right)$ of Section 6.2, that is an intersection of maximal isotropic subgroups. 
Proposition 6.1. Let $E$ be an elliptic curve over a global field $k$. Let $p$ be a prime and let $e \in \mathbb{Z}_{\geq 0}$. If char $k \neq p$, suppose that the image $G$ of $G_{k} \rightarrow$ Aut $E\left[p^{e}\right] \simeq \mathrm{GL}_{2}\left(\mathbb{Z} / p^{e} \mathbb{Z}\right)$ contains $\mathrm{SL}_{2}\left(\mathbb{Z} / p^{e} \mathbb{Z}\right)$. If char $k=p$, suppose that the image $G$ of $G_{k} \rightarrow$ Aut $E\left[p^{e}\right]\left(k^{\mathrm{sep}}\right)$ is cyclic. Then $\amalg^{1}\left(k, E\left[p^{e}\right]\right)=0$.

Remark 6.2. For each $k$, the hypothesis of Proposition 6.1 holds for $100 \%$ of elliptic curves over $k$, as we now explain. If char $k \neq p$, then the result follows from the Hilbert irreducibility theorem. If char $k=p$, then $E\left[p^{e}\right]\left(k^{\mathrm{sep}}\right)$ is cyclic of order $p^{f}$ for some $f \leq e$, and its automorphism group is $\left(\mathbb{Z} / p^{f} \mathbb{Z}\right)^{\times}$; if moreover, $p>2$ or $e \leq 2$, then $\left(\mathbb{Z} / p^{f} \mathbb{Z}\right)^{\times}$is cyclic, so the hypothesis holds for all elliptic curves over $k$. Finally, if char $k=2$, then an explicit calculation with Weierstrass equations shows that $E[2]\left(k^{\mathrm{sep}}\right)=0$ for $100 \%$ of $E$ (i.e., in the ordinary case, the unique nontrivial point of $E[2](\bar{k})$ is usually defined over an inseparable extension); for such $E$, we have $E\left[2^{e}\right]\left(k^{\text {sep }}\right)=0$.

Before proving Proposition 6.1, we introduce some more definitions and prove a few basic facts. For any finite group $G$ and $G$-module $M$, define

$$
\mathrm{H}_{\text {cyc }}^{1}(G, M):=\bigcap_{\text {cyclic } H \leq G} \operatorname{ker}\left(\mathrm{H}^{1}(G, M) \rightarrow \mathrm{H}^{1}(H, M)\right),
$$

which, like $\mathrm{H}^{1}(G, M)$, is contravariant in $G$ and covariant in $M$. For any Galois extension $L / k$ and $\operatorname{Gal}(L / k)$-module $M$, define

$$
\amalg^{1}(L / k, M):=\operatorname{ker}\left(\mathrm{H}^{1}(\operatorname{Gal}(L / k), M) \rightarrow \prod_{v \in \Omega} \mathrm{H}^{1}\left(\operatorname{Gal}\left(L_{w} / k_{v}\right), M\right)\right),
$$

where $\operatorname{Gal}\left(L_{w} / k_{v}\right)$ is a decomposition group associated to a chosen place $w$ of $L$ above $v$; since the conjugation action $\operatorname{Gal}(L / k)$ on itself induces the identity on $\mathrm{H}^{1}(\operatorname{Gal}(L / k), M)$, it does not matter which $w$ is chosen, and we could alternatively take the kernel of the map to the product over all $w$ instead of using only one above each $v$.

Lemma 6.3 (cf. [BPS13, Proposition 8.3]).

(a) Let $G$ be a finite group acting trivially on an abelian group $M$. Then $\mathrm{H}_{\text {cyc }}^{1}(G, M)=0$.

(b) If $L / k$ is a finite Galois extension with Galois group $G$, and $M$ is a $G$-module, then $\amalg^{1}(L / k, M) \subseteq \mathrm{H}_{\text {cyc }}^{1}(G, M)$.

(c) If $L / k$ is a Galois extension with Galois group $G$, and $G$ acts trivially on an abelian group $M$, then $\amalg^{1}(L / k, M)=0$. 
(d) If $L / k$ is a finite Galois extension, and $M$ is a $\operatorname{Gal}(L / k)$-module, and $L^{\prime} / k$ is a larger Galois extension (so $\operatorname{Gal}\left(L^{\prime} / L\right)$ acts trivially on $\left.M\right)$, then inflation induces an isomorphism $\amalg^{1}(L / k, M) \stackrel{\sim}{\rightarrow} \amalg^{1}\left(L^{\prime} / k, M\right)$.

(e) If $L^{\prime} / k$ is a Galois extension, and $M$ is a finite $\operatorname{Gal}\left(L^{\prime} / k\right)$-module, and $G$ is the image of $\operatorname{Gal}\left(L^{\prime} / k\right) \rightarrow$ Aut $M$, then $\amalg^{1}\left(L^{\prime} / k, M\right)$ is isomorphic to a subgroup of $\mathrm{H}_{\mathrm{cyc}}^{1}(G, M)$.

Proof.

(a) A homomorphism $G \rightarrow M$ that restricts to 0 on each cyclic subgroup of $G$ is 0 .

(b) By the Chebotarev density theorem, each cyclic subgroup of $G$ arises as a decomposition subgroup.

(c) If $L / k$ is finite, this follows from (a) and (b). The general case follows by taking a direct limit.

(d) From the inflation-restriction sequence

$$
0 \rightarrow \mathrm{H}^{1}(\operatorname{Gal}(L / k), M) \rightarrow \mathrm{H}^{1}\left(\operatorname{Gal}\left(L^{\prime} / k\right), M\right) \rightarrow \mathrm{H}^{1}\left(\operatorname{Gal}\left(L^{\prime} / L\right), M\right)
$$

mapping to its local analogues, we obtain an exact sequence

$$
0 \rightarrow \amalg^{1}(L / k, M) \rightarrow \amalg^{1}\left(L^{\prime} / k, M\right) \rightarrow \amalg^{1}\left(L^{\prime} / L, M\right) .
$$

The last term is 0 by (c).

(e) The quotient $G$ of $\operatorname{Gal}\left(L^{\prime} / k\right)$ is $\operatorname{Gal}(L / k)$ for a finite Galois extension $L / k$. Apply (d) and then (b).

Proof of Proposition 6.1 for char $k \neq p$. The case $e=1$ is [PR12, Proposition 3.3(e)], so assume $e \geq 2$. Let $S_{e}:=\mathrm{SL}_{2}\left(\mathbb{Z} / p^{e} \mathbb{Z}\right)$. Let $M:=E\left[p^{e}\right] \simeq$ $\left(\mathbb{Z} / p^{e} \mathbb{Z}\right)^{2}$. By Lemma $6.3(\mathrm{e}), \amalg^{1}(k, M)$ is isomorphic to a subgroup of $\mathrm{H}_{\text {cyc }}^{1}(G, M)$. The invariant subgroup $M^{S_{e}}$ is 0 , so the inflation-restriction sequence for $S_{e} \leq G$ shows that $\mathrm{H}_{\text {cyc }}^{1}(G, M) \rightarrow \mathrm{H}_{\text {cyc }}^{1}\left(S_{e}, M\right)$ is injective. It remains to show that $\mathrm{H}_{\text {cyc }}^{1}\left(S_{e}, M\right)=0$.

The inflation-restriction sequence associated to the central subgroup $\{ \pm 1\} \leq S_{e}$ is

$$
0 \longrightarrow \mathrm{H}^{1}\left(S_{e} /\{ \pm 1\}, M[2]\right) \stackrel{\inf }{\longrightarrow} \mathrm{H}^{1}\left(S_{e}, M\right) \longrightarrow \mathrm{H}^{1}(\{ \pm 1\}, M)^{S_{e}}
$$

If $p$ is odd, $M[2]=0$ and $\mathrm{H}^{1}(\{ \pm 1\}, M)=0$ (killed by both 2 and $p$ ), so $\mathrm{H}^{1}\left(S_{e}, M\right)=0$. 
So assume that $p=2$. Then $\mathrm{H}^{1}(\{ \pm 1\}, M) \simeq(\mathbb{Z} / 2 \mathbb{Z})^{2}$, on which $S_{e}$ acts through $S_{1}$ in the standard way, so $\mathrm{H}^{1}(\{ \pm 1\}, M)^{S_{e}}=0$, so the map inf in (13) is an isomorphism. The map inf factors as

$$
\mathrm{H}^{1}\left(S_{e} /\{ \pm 1\}, M[2]\right) \longrightarrow \mathrm{H}^{1}\left(S_{e}, M[2]\right) \longrightarrow \mathrm{H}^{1}\left(S_{e}, M\right),
$$

so the second map is surjective. It is also injective, since $\mathrm{H}^{0}\left(S_{e}, 2 M\right)=0$. Thus $\mathrm{H}^{1}\left(S_{e}, M[2]\right) \simeq \mathrm{H}^{1}\left(S_{e}, M\right)$.

Define a filtration

$$
\{1\} \leq \Gamma_{e-1} \leq \cdots \leq \Gamma_{2} \leq \Gamma_{1} \leq S_{e}
$$

by $\Gamma_{m}:=\operatorname{ker}\left(S_{e} \rightarrow S_{m}\right)$. We prove by induction on $e$ that the inclusion $\Gamma_{1}^{2}\left[\Gamma_{1}, \Gamma_{1}\right] \leq \Gamma_{2}$ is an equality. We check the cases $e=2$ and $e=3$ by hand. For $e \geq 4$, every element of $\Gamma_{e-1}$ is represented by $1+2^{e-1} A$ for some trace-0 integer matrix $A$, and is the square of $1+2^{e-2} A \in \Gamma_{e-2} \leq \Gamma_{1}$; now apply the inductive hypothesis to $S_{e-1}=S_{e} / \Gamma_{e-1}$.

The previous paragraph shows that $\Gamma_{2}$ is contained in the kernel of every homomorphism $\Gamma_{1} \rightarrow \mathbb{Z} / 2 \mathbb{Z}$. Thus the restriction map $\mathrm{H}^{1}\left(\Gamma_{1}, M[2]\right) \rightarrow$ $\mathrm{H}^{1}\left(\Gamma_{2}, M[2]\right)$ is 0 (the actions are trivial). Consider the maps $\alpha$ and $\beta$ in the inflation-restriction sequence

$$
0 \longrightarrow \mathrm{H}^{1}\left(S_{2}, M[2]\right) \stackrel{\alpha}{\longrightarrow} \mathrm{H}^{1}\left(S_{e}, M[2]\right) \stackrel{\beta}{\longrightarrow} \mathrm{H}^{1}\left(\Gamma_{2}, M[2]\right) .
$$

Since $\beta$ factors through $\mathrm{H}^{1}\left(\Gamma_{1}, M[2]\right) \rightarrow \mathrm{H}^{1}\left(\Gamma_{2}, M[2]\right)$, we have $\beta=0$, and $\alpha$ is an isomorphism. Let $U_{e} \leq S_{e}$ be the subgroup of unipotent upper triangular matrices. The horizontal maps in the bottom row of the commutative diagram

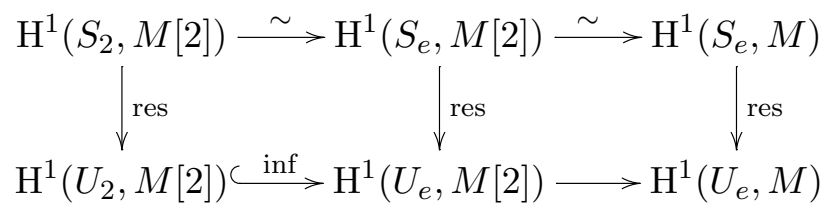

are injective (for the second map, observe that $M^{U_{e}} \stackrel{2}{\rightarrow}(2 M)^{U_{e}}$ is surjective). Direct calculation shows that the left vertical map is injective too (in fact, it is an isomorphism between groups of order 2). So the right vertical map is injective. In particular, $\mathrm{H}_{\text {cyc }}^{1}\left(S_{e}, M\right)=0$, since $U_{e}$ is cyclic.

The following two lemmas will be used in the proof of the char $k=p$ case of Proposition 6.1. 
Lemma 6.4. Let $k$ be a field of characteristic p. Let $E$ be an elliptic curve over $k$.

(a) If $E$ is ordinary, then for any $e \in \mathbb{Z}_{\geq 0}$ there is an exact sequence

$$
0 \rightarrow M^{\vee} \rightarrow E\left[p^{e}\right] \rightarrow M \rightarrow 0
$$

where $M$ is a finite étale group scheme of order $p^{e}$ and $M^{\vee}$ is its Cartier dual.

(b) If $E$ is supersingular, then $E\left[p^{e}\right]$ is an iterated extension of copies of $\alpha_{p}$.

Proof. Let $F: E \rightarrow E^{\prime}$ be the $p^{e}$-Frobenius morphism, and let $V: E^{\prime} \rightarrow E$ be its dual. Then $F$ is surjective and $V F=p^{e}$, so there is an exact sequence

$$
0 \rightarrow \operatorname{ker} F \rightarrow E\left[p^{e}\right] \rightarrow \operatorname{ker} V \rightarrow 0
$$

Moreover, $\operatorname{ker} F$ is the Cartier dual of $\operatorname{ker} V$, by [Mum70, III.15, Theorem 1] (the proof there works over any field).

(a) If $E$ is ordinary, then $\operatorname{ker} V$ is a finite étale group scheme of order $\operatorname{deg} V=p^{e}$.

(b) Suppose that $E$ is supersingular. The group scheme $E\left[p^{e}\right]$ is an iterated extension of copies of $E[p]$, so we may reduce to the case $e=1$. If $e=1$, then $\operatorname{ker} F$ and ker $V$ are isomorphic to $\alpha_{p}$ : over an algebraically closed field, this is well known [Oor66, II.15.5], and it follows over any field of characteristic $p$ since the twists of $\alpha_{p}$ are classified by $\mathrm{H}^{1}\left(k, \mathbf{A u t} \alpha_{p}\right)=$ $\mathrm{H}^{1}\left(k, \mathbb{G}_{m}\right)=0$.

Lemma 6.5. Let $k$ be a global field of characteristic $p$. Let $M$ be a finite commutative group scheme over $k$ that is an iterated extension of copies of $\mu_{p}$ and $\alpha_{p}$. If $v \in \Omega$, then $\mathrm{H}^{1}(k, M) \rightarrow \mathrm{H}^{1}\left(k_{v}, M\right)$ is injective. In particular, $\amalg^{1}(k, M)=0$.

Proof. When $M=\mu_{p}$, Hilbert's theorem 90 implies that $\mathrm{H}^{1}(k, M) \rightarrow$ $\mathrm{H}^{1}\left(k_{v}, M\right)$ is $k^{\times} / k^{\times p} \rightarrow k_{v}^{\times} / k_{v}^{\times p}$. Similarly, when $M=\alpha_{p}$, it is the homomorphism of additive groups $k / k^{p} \rightarrow k_{v} / k_{v}^{p}$. Both homomorphisms are injective, by [PV10, Lemma 3.1].

If $0 \rightarrow M^{\prime} \rightarrow M \rightarrow M^{\prime \prime} \rightarrow 0$ is an extension of group schemes as in the statement, and the result holds for $M^{\prime}$ and $M^{\prime \prime}$, then it holds for $M$ too (this uses injectivity of $\mathrm{H}^{1}\left(k_{v}, M^{\prime}\right) \rightarrow \mathrm{H}^{1}\left(k_{v}, M\right)$, which follows since $\left.\mathrm{H}^{0}\left(k_{v}, M^{\prime \prime}\right)=0\right)$. So the general case follows by induction on \# $M$. 
Proof of Proposition 6.1 for char $k=p$.

Case 1: $E$ is supersingular. Combine Lemmas 6.4(b) and 6.5.

Case 2: $E$ is ordinary. Let $M$ be as in Lemma 6.4(a). Let $N=E\left[p^{e}\right]\left(k^{\mathrm{sep}}\right)$, which injects into $M\left(k^{\text {sep }}\right)$ under the map induced by (14). Let $L$ be the splitting field of $M$. Thus $L$ is a Galois extension of $k$ and the image of $\operatorname{Gal}(L / k) \rightarrow$ Aut $N$ is $G$. We now break into subcases.

Case 2a: $L=k$. Then (14) has the form

$$
0 \rightarrow \mu_{p^{e}} \rightarrow E\left[p^{e}\right] \rightarrow \mathbb{Z} / p^{e} \mathbb{Z} \rightarrow 0 .
$$

By Lemma 6.3(c), $\amalg^{1}\left(k, \mathbb{Z} / p^{e} \mathbb{Z}\right)=0$, so any $\xi \in \amalg^{1}\left(k, E\left[p^{e}\right]\right)$ must come from an element $\eta \in \mathrm{H}^{1}\left(k, \mu_{p^{e}}\right)$. Pick any $v \in \Omega$. The middle vertical map in the commutative diagram

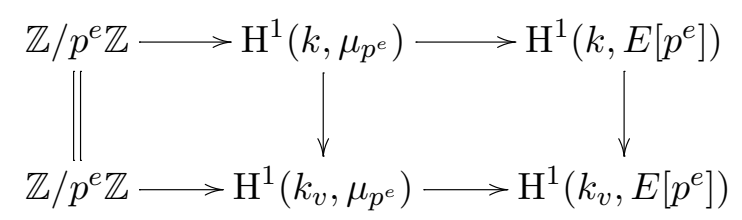

is injective by Lemma 6.5 , and a diagram chase shows that $\eta$ comes from an element of $\mathbb{Z} / p^{e} \mathbb{Z}$. Thus $\xi=0$.

Case 2b: $L$ is general. By definition of $L$, we have $E\left[p^{e}\right](L)=N$. For any place $w$ of $L$, every element of $L_{w}$ that is algebraic over $L$ is actually separable over $L$ [PV10, Lemma 3.1], so $E\left[p^{e}\right]\left(L_{w}\right)=N$ too. By Case 2a, $\amalg^{1}\left(L, E\left[p^{e}\right]\right)=0$. Because of the (fppf) inflation-restriction sequence

$$
0 \rightarrow \mathrm{H}^{1}(\operatorname{Gal}(L / k), N) \rightarrow \mathrm{H}^{1}\left(k, E\left[p^{e}\right]\right) \rightarrow \mathrm{H}^{1}\left(L, E\left[p^{e}\right]\right),
$$

which maps to its analogue for each extension $L_{w} / k_{v}$ of local fields, we have $\amalg^{1}\left(k, E\left[p^{e}\right]\right) \simeq \amalg^{1}(\operatorname{Gal}(L / k), N)$. By Lemma 6.3(e), the latter is isomorphic to a subgroup of $\mathrm{H}_{\text {cyc }}^{1}(G, N)$, which is trivial since $G$ is cyclic by assumption.

Remark 6.6. In Proposition 6.1, when $p=2$ and $e=3$, the hypothesis that the image of $G_{k} \rightarrow$ Aut $E\left[p^{e}\right]\left(k^{\mathrm{sep}}\right)$ is cyclic can fail (but only for $0 \%$ of $E \in \mathscr{E}$, as explained in Remark 6.2). The last line of the proof above cannot be immediately extended to the case in which the image is non-cyclic, because one can check that $\mathrm{H}_{\text {cyc }}^{1}\left((\mathbb{Z} / 8 \mathbb{Z})^{\times}, \mathbb{Z} / 8 \mathbb{Z}\right) \neq 0$ for the standard nontrivial action. The conclusion of Proposition 6.1 might still hold, however. 


\subsection{Intersection of maximal isotropic subgroups}

For nonarchimedean $v$, let $\mathcal{O}_{v}$ be the valuation ring in $k_{v}$. Let

$$
\mathbf{A}=\prod_{v \in \Omega}^{\prime}\left(k_{v}, \mathcal{O}_{v}\right)
$$

be the adele ring of $k$. Suppose that $E, k$, and $p^{e}$ satisfy the hypothesis of Proposition 6.1, so that $\amalg^{1}\left(k, E\left[p^{e}\right]\right)=0$. Then [PR12, Theorem 4.14] applied with $\lambda: A \rightarrow \widetilde{A}$ being $\left[p^{e}\right]: E \rightarrow E$ shows that $\operatorname{Sel}_{p^{e}} E$ is isomorphic to the intersection of two maximal isotropic subgroups of

$$
\begin{aligned}
\mathrm{H}^{1}\left(\mathbf{A}, E\left[p^{e}\right]\right) & :=\prod_{v \in \Omega}^{\prime}\left(\mathrm{H}^{1}\left(k_{v}, E\left[p^{e}\right]\right), \mathrm{H}^{1}\left(\mathcal{O}_{v}, E\left[p^{e}\right]\right)\right) \\
& \simeq \prod_{v \in \Omega}^{\prime}\left(\mathrm{H}^{1}\left(k_{v}, E\left[p^{e}\right]\right), \frac{E\left(k_{v}\right)}{p^{e} E\left(k_{v}\right)}\right),
\end{aligned}
$$

namely the images of $E(\mathbf{A}) / p^{e} E(\mathbf{A})=\prod_{v} E\left(k_{v}\right) / p^{e} E\left(k_{v}\right)$ and $\mathrm{H}^{1}\left(k, E\left[p^{e}\right]\right)$.

\subsection{Direct summands}

It is natural to ask whether these images are direct summands, given that we modeled $\mathrm{Sel}_{p^{e}} E$ by an intersection of direct summands. Corollary 6.8 below shows that at least the first of these images is a direct summand.

Proposition 6.7. Let $E$ be an abelian variety over an arbitrary field $k$. Let $n \in \mathbb{Z}_{>0}$. Then the image of the coboundary map $E(k) / n E(k) \stackrel{\delta}{\rightarrow} \mathrm{H}^{1}(k, E[n])$ is a direct summand of $\mathrm{H}^{1}(k, E[n])$.

Proof. (We thank Bart de Smit and Christopher Skinner for ideas used in this proof.) For each $m \mid n$, the commutative diagram

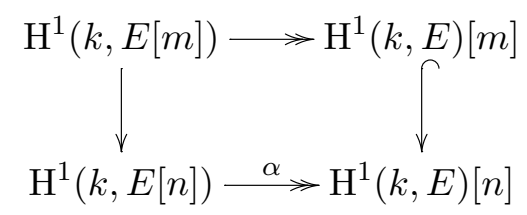

shows that any order $m$ element of $\mathrm{H}^{1}(k, E)[n]$ lifts to an order $m$ element of $\mathrm{H}^{1}(k, E[n])$ under the surjection $\alpha$ in the diagram. Any $\mathbb{Z} / n \mathbb{Z}$-module is a 
direct sum of cyclic groups [Prü23, §17], [Bae35, pp. 274-275]; applying this to $\mathrm{H}^{1}(k, E)[n]$ and using the previous sentence shows that $\alpha$ is split. Finally, $\operatorname{ker}(\alpha)=\operatorname{im}(\delta)$.

Corollary 6.8. Let $E$ be an abelian variety over a global field $k$. Let $n \in \mathbb{Z}_{>0}$. Then the image of $E(\mathbf{A}) / n E(\mathbf{A}) \stackrel{\delta}{\rightarrow} \mathrm{H}^{1}(\mathbf{A}, E[n])$ is a direct summand of $\mathrm{H}^{1}(\mathbf{A}, E[n])$.

Proof. Proposition 6.7 yields a complement $C_{v}$ of the subgroup $E\left(k_{v}\right) / n E\left(k_{v}\right)$ in $\mathrm{H}^{1}\left(k_{v}, E[n]\right)$. Then $\bigoplus_{v \in \Omega} C_{v}$ is a complement of the subgroup $E(\mathbf{A}) / n E(\mathbf{A})$ in $\mathrm{H}^{1}(\mathbf{A}, E[n])$.

Is the other subgroup, the image of $\mathrm{H}^{1}(k, E[n]) \rightarrow \mathrm{H}^{1}(\mathbf{A}, E[n])$, a direct summand too? Lemma 6.10 below gives a positive answer for some elliptic curves. Although it applies only to $0 \%$ of $E \in \mathscr{E}$, it may be that the answer is positive for all $E$. We conjecture at least the following.

Conjecture 6.9. Fix a global field $k$ and $n \geq 1$. The image of $\mathrm{H}^{1}(k, E[n]) \rightarrow$ $\mathrm{H}^{1}(\mathbf{A}, E[n])$ is a direct summand for $100 \%$ of $E \in \mathscr{E}$.

Lemma 6.10. If char $k \nmid n$ and the action of $G_{k}$ on $E[n]$ is trivial, then the image of $\mathrm{H}^{1}(k, E[n]) \rightarrow \mathrm{H}^{1}(\mathbf{A}, E[n])$ is a direct summand.

Proof. We have $E[n] \simeq \mu_{n} \times \mu_{n}$, so we must show that the image of $k^{\times} / k^{\times n} \rightarrow \mathbf{A}^{\times} / \mathbf{A}^{\times n}$ is a direct summand. By Lemma 6.11 below, it is enough to show that $k^{\times} / k^{\times m} \rightarrow \mathbf{A}^{\times} / \mathbf{A}^{\times m}$ is injective for each $m \mid n$. This "local-global principle for $m^{\text {th }}$ powers" is a well known consequence of the Chebotarev density theorem.

Lemma 6.11. Let $n \in \mathbb{Z}_{>0}$. Let $\delta: A \rightarrow B$ be a homomorphism of $\mathbb{Z} / n \mathbb{Z}$ modules such that the induced morphism $A / m A \rightarrow B / m B$ is injective for every $m \mid n$. Then $\delta(A)$ is a direct summand of $B$.

Proof. (We thank Bart de Smit for this proof.) Taking $m=n$ shows that $\delta$ is injective, so it fits into a short exact sequence

$$
0 \rightarrow A \stackrel{\delta}{\rightarrow} B \rightarrow C \rightarrow 0
$$

of $\mathbb{Z} / n \mathbb{Z}$-modules. Write $C$ as a direct sum of cyclic groups $C_{i}$. For each $m$, the hypothesis together with the snake lemma shows that $B[m] \rightarrow C[m]$ is surjective. Thus we can construct a splitting of the surjection $B \rightarrow C$, by lifting a generator of each $C_{i}$ to an element of $B$ of the same order. 
Remark 6.12. Suppose that for each $n$ we sample $Z$ and $W$ from a distribution on maximal isotropic subgroups of $\left(\mathbb{Z} / p^{e} \mathbb{Z}\right)^{2 n}$ that is not necessarily supported on direct summands, but still invariant under $\mathrm{O}_{2 n}\left(\mathbb{Z} / p^{e} \mathbb{Z}\right)$. If

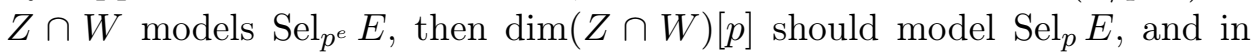
particular should have the distribution predicted by and justified by [PR12]. We will show that this happens only if the probability of $Z$ and $W$ being direct summands of $\left(\mathbb{Z} / p^{e} \mathbb{Z}\right)^{2 n}$ tends to 1 as $n \rightarrow \infty$.

If $(Z \cap W)[p]=0$, then $Z \cap W=0$, so the homomorphism $Z \oplus W \rightarrow$ $\left(\mathbb{Z} / p^{e} \mathbb{Z}\right)^{2 n}$ between groups of equal size is an isomorphism; i.e., $Z$ and $W$ are direct summands. Therefore

$\operatorname{Prob}((Z \cap W)[p]=0) \leq \operatorname{Prob}(Z, W$ are direct summands $)$ $\times \operatorname{Prob}(Z \cap W=0 \mid Z, W$ are direct summands $)$.

But $\lim _{n \rightarrow \infty} \operatorname{Prob}(Z \cap W=0 \mid Z, W$ are direct summands $)$ equals the desired limiting value of $\operatorname{Prob}((Z \cap W)[p]=0)$, which is nonzero, so

$\operatorname{Prob}(Z, W$ are direct summands $) \rightarrow 1$.

Remark 6.12 may be viewed as indirect evidence for Conjecture 6.9.

\subsection{Freeness of the ambient group}

So far in Section 6 we have modeled the $\mathbb{Z} / p^{e} \mathbb{Z}$-module $\mathrm{H}^{1}\left(\mathbf{A}, E\left[p^{e}\right]\right)$ by the free module $\left(\mathbb{Z} / p^{e} \mathbb{Z}\right)^{2 n}$. But the following shows that in reality, $\mathrm{H}^{1}\left(\mathbf{A}, E\left[p^{e}\right]\right)$ is almost never free.

Proposition 6.13. Fix a prime $p$ and integer $e \geq 2$. Fix a global field $k$ such that char $k \neq p$. Then for $100 \%$ of elliptic curves $E$ over $k$, the $\mathbb{Z} / p^{e} \mathbb{Z}$-module $\mathrm{H}^{1}\left(\mathbf{A}, E\left[p^{e}\right]\right)$ is not free.

Proof. By the Hilbert irreducibility theorem, for $100 \%$ of elliptic curves $E$, the homomorphism $\rho: G_{k} \rightarrow$ Aut $E\left[p^{e}\right] \simeq \mathrm{GL}_{2}\left(\mathbb{Z} / p^{e} \mathbb{Z}\right)$ is surjective. Consider such an $E$. The Chebotarev density theorem yields a place $v$ of good reduction not dividing $p$ such that $\rho\left(\operatorname{Frob}_{v}\right)=\left(\begin{array}{cc}1+p & 0 \\ 0 & 1+p\end{array}\right)$; then $E\left(k_{v}\right)\left[p^{e}\right] \simeq$ $(\mathbb{Z} / p \mathbb{Z})^{2}$. This group is a direct summand of $E\left(k_{v}\right)$, so $E\left(k_{v}\right) / p^{e} E\left(k_{v}\right)$ is not a free $\mathbb{Z} / p^{e} \mathbb{Z}$-module. By Proposition $6.7, E\left(k_{v}\right) / p^{e} E\left(k_{v}\right)$ is a direct summand of $\mathrm{H}^{1}\left(k_{v}, E\left[p^{e}\right]\right)$, which is a direct summand of $\mathrm{H}^{1}\left(\mathbf{A}, E\left[p^{e}\right]\right)$, so the latter is not free. 
Question 6.14. Can we develop a more sophisticated model in which we start with a compatible system consisting of a quadratic form on a non-free $\mathbb{Z} / p^{e} \mathbb{Z}$-module for each $e$ ?

Given the compatibility of our model with known theorems and conjectures, we expect that incorporating non-freeness into the model would not change the distribution constructed in Section 1.2.

\section{Acknowledgements}

We thank the referee for reading our paper carefully and for making many insightful comments. We also thank Kęstutis Česnavičius, Bart de Smit, Christophe Delaunay, and Christopher Skinner for helpful comments. This research was begun during the "Arithmetic Statistics" semester at the Mathematical Sciences Research Institute, and continued during the "Cohen-Lenstra heuristics for class groups" workshop at the American Institute of Mathematics, the 2012 Canadian Number Theory Association meeting at the University of Lethbridge, the Centre Interfacultaire Bernoulli semester on "Rational points and algebraic cycles", the 2013 "Explicit methods in number theory" workshop at the Mathematisches Forschungsinstitut Oberwolfach, the "Rational points 2013" workshop at Schloss Thurnau, and the "Counting arithmetic objects" workshop at the Centre de Recherches Mathématiques in Montreal.

\section{References}

[Bae35] Reinhold Baer, Der Kern, ein charakteristische Untergruppe, Compositio Math. 1 (1935), 254-283.

[BS15a] Manjul Bhargava and Arul Shankar, Binary quartic forms having bounded invariants, and the boundedness of the average rank of elliptic curves, Ann. of Math. (2) 181 (2015), no. 1, 191-242, DOI 10.4007/annals.2015.181.1.3. MR3272925

[BS15b] _ Ternary cubic forms having bounded invariants, and the existence of a positive proportion of elliptic curves having rank 0, Ann. of Math. (2) 181 (2015), no. 2, 587-621, DOI 10.4007/annals.2015.181.2.4. MR3275847

[BS13a] _ The average number of elements in the 4-Selmer groups of elliptic curves is 7, December 27, 2013. Preprint, arXiv:1312.7333v1.

[BS13b] , The average size of the 5-Selmer group of elliptic curves is 6, and the average rank is less than 1, December 30, 2013. Preprint, arXiv: $1312.7859 \mathrm{v} 1$. 
[Bou98] Nicolas Bourbaki, Commutative algebra. Chapters 1-7, Elements of Mathematics (Berlin), Springer-Verlag, Berlin, 1998. Translated from the French; Reprint of the 1989 English translation. MR1727221 (2001g:13001)

[BPS13] Nils Bruin, Bjorn Poonen, and Michael Stoll, Generalized explicit descent and its application to curves of genus 3, December 11, 2013. Preprint, arXiv:1205.4456v2.

[CL84] H. Cohen and H. W. Lenstra Jr., Heuristics on class groups of number fields, Number theory, Noordwijkerhout 1983 (Noordwijkerhout, 1983), Lecture Notes in Math., vol. 1068, Springer, Berlin, 1984, pp. 33-62, DOI 10.1007/BFb0099440, (to appear in print). MR756082 (85j:11144)

[Del01] Christophe Delaunay, Heuristics on Tate-Shafarevitch groups of elliptic curves defined over $\mathbb{Q}$, Experiment. Math. 10 (2001), no. 2, 191-196. MR1837670 (2003a:11065)

[Del07] Heuristics on class groups and on Tate-Shafarevich groups: the magic of the Cohen-Lenstra heuristics, Ranks of elliptic curves and random matrix theory, London Math. Soc. Lecture Note Ser., vol. 341, Cambridge Univ. Press, Cambridge, 2007, pp. 323-340. MR2322355 (2008i:11089)

[DJ14a] Christophe Delaunay and Frédéric Jouhet, $p^{\ell}$-torsion points in finite abelian groups and combinatorial identities, Adv. Math. 258 (2014), 13-45, DOI 10.1016/j.aim.2014.02.033. MR3190422

[DJ14b] , The Cohen-Lenstra heuristics, moments and $p^{j}$-ranks of some groups, Acta Arith. 164 (2014), no. 3, 245-263, DOI 10.4064/aa164-3-3. MR3238116

[EGA I] A. Grothendieck and J. A. Dieudonné, Eléments de géométrie algébrique. I 166 (1971), ix +466 (French). MR3075000

[Eul48] Leonhard Euler, Introductio in analysin infinitorum. Tomus primus, Marcum-Michaelum Bousquet \& Socios, Lausanne, 1748; English transl., Introduction to analysis of the infinite. Book I, Springer-Verlag, New York, 1988. Translated from the Latin and with an introduction by John D. Blanton. MR961255 (89g:01067).

[FX12] Keqin Feng and Maosheng Xiong, On Selmer groups and TateShafarevich groups for elliptic curves $y^{2}=x^{3}-n^{3}$, Mathematika $\mathbf{5 8}$ (2012), no. 2, 236-274, DOI 10.1112/S0025579312000046. MR2965971

[FW89] Eduardo Friedman and Lawrence C. Washington, On the distribution of divisor class groups of curves over a finite field, Théorie des nombres (Quebec, PQ, 1987), de Gruyter, Berlin, 1989, pp. 227-239. MR1024565 (91e:11138)

[Gol79] Dorian Goldfeld, Conjectures on elliptic curves over quadratic fields, Number theory, Carbondale 1979 (Proc. Southern Illinois Conf., Southern Illinois Univ., Carbondale, Ill., 1979), Lecture Notes in Math., vol. 751, Springer, Berlin, 1979, pp. 108-118. MR564926 (81i:12014)

[Igu00] Jun-ichi Igusa, An introduction to the theory of local zeta functions, AMS/IP Studies in Advanced Mathematics, vol. 14, American Mathematical Society, Providence, RI, 2000. MR1743467 (2001j:11112) 
[KM95] S. Kamienny and B. Mazur, Rational torsion of prime order in elliptic curves over number fields, Astérisque 228 (1995), 3, 81-100. With an appendix by A. Granville; Columbia University Number Theory Seminar (New York, 1992). MR1330929 (96c:11058)

[KS99a] Nicholas M. Katz and Peter Sarnak, Random matrices, Frobenius eigenvalues, and monodromy, American Mathematical Society Colloquium Publications, vol. 45, American Mathematical Society, Providence, RI, 1999. MR 2000b:11070

[KS99b] Zeroes of zeta functions and symmetry, Bull. Amer. Math. Soc. (N.S.) 36 (1999), no. 1, 1-26, DOI 10.1090/S0273-0979-99-00766-1. MR1640151 (2000f:11114)

[KLO15] Zev Klagsbrun and Robert J. Lemke Oliver, The distribution of 2-Selmer ranks of quadratic twists of elliptic curves with partial two-torsion, Mathematika FirstView (2015), 1-12, DOI 10.1112/S0025579315000121. Available at http://journals.cambridge. org/article_S0025579315000121.

[Lev68] Martin Levin, On the group of rational points on elliptic curves over function fields, Amer. J. Math. 90 (1968), 456-462. MR0230723 (37 \#6283)

[Maz77] B. Mazur, Modular curves and the Eisenstein ideal, Inst. Hautes Études Sci. Publ. Math. 47 (1977), 33-186 (1978). MR488287 (80c:14015)

[Mer96] Loïc Merel, Bornes pour la torsion des courbes elliptiques sur les corps de nombres, Invent. Math. 124 (1996), no. 1-3, 437-449 (French). MR1369424 (96i:11057)

[Mum70] David Mumford, Abelian varieties, Tata Institute of Fundamental Research Studies in Mathematics, No. 5, Published for the Tata Institute of Fundamental Research, Bombay, 1970. MR0282985 (44 \#219)

[Oes82] Joseph Oesterlé, Réduction modulo $p^{n}$ des sous-ensembles analytiques fermés de $\mathbf{Z}_{p}^{N}$, Invent. Math. 66 (1982), no. 2, 325-341, DOI 10.1007/BF01389398 (French). MR656627 (83j:12014)

[Oor66] F. Oort, Commutative group schemes, Lecture Notes in Mathematics, vol. 15, Springer-Verlag, Berlin, 1966. MR0213365 (35 \#4229)

[Poo13] Bjorn Poonen, Average rank of elliptic curves /after Manjul Bhargava and Arul Shankar], Astérisque 352 (2013), Exp. No. 1049, viii, 187-204. Séminaire Bourbaki. Vol. 2011/2012. Exposés 1043-1058. MR3087347

[PR12] Bjorn Poonen and Eric Rains, Random maximal isotropic subspaces and Selmer groups, J. Amer. Math. Soc. 25 (2012), no. 1, 245-269, DOI 10.1090/S0894-0347-2011-00710-8. MR2833483

[PV10] Bjorn Poonen and José Felipe Voloch, The Brauer-Manin obstruction for subvarieties of abelian varieties over function fields, Ann. of Math. (2) 171 (2010), no. 1, 511-532, DOI 10.4007/annals.2010.171.511. MR2630046 (2011j:14048)

[Prü23] Heinz Prüfer, Untersuchungen über die Zerlegbarkeit der abzählbaren primären Abelschen Gruppen, Math. Z. 17 (1923), no. 1, 35-61, DOI 10.1007/BF01504333 (German). MR1544601 
[Ser81] Jean-Pierre Serre, Quelques applications du théorème de densité de Chebotarev, Inst. Hautes Études Sci. Publ. Math. 54 (1981), 323-401 (French). MR644559 (83k:12011)

[SGA 7II] Groupes de monodromie en géométrie algébrique. II, Lecture Notes in Mathematics, Vol. 340, Springer-Verlag, Berlin, 1973 (French). Séminaire de Géométrie Algébrique du Bois-Marie 1967-1969 (SGA 7 II); Dirigé par P. Deligne et N. Katz. MR0354657 (50 \#7135)

[VE10] Akshay Venkatesh and Jordan S. Ellenberg, Statistics of number fields and function fields, Proceedings of the International Congress of Mathematicians. Volume II, Hindustan Book Agency, New Delhi, 2010, pp. 383-402. MR2827801 (2012h:11160)

[XZ08] Maosheng Xiong and Alexandru Zaharescu, Distribution of Selmer groups of quadratic twists of a family of elliptic curves, Adv. Math. 219 (2008), no. 2, 523-553, DOI 10.1016/j.aim.2008.05.005. MR2435648 (2009e:11113)

[XZ09]_ Selmer groups and Tate-Shafarevich groups for the congruent number problem, Comment. Math. Helv. 84 (2009), no. 1, 21-56, DOI 10.4171/CMH/151. MR2466074 (2010c:11068)

[Yu05] Gang Yu, Average size of 2-Selmer groups of elliptic curves. II, Acta Arith. 117 (2005), no. 1, 1-33, DOI 10.4064/aa117-1-1. MR2110501 (2006b:11054)

Manjul Bhargava

Department of Mathematics

Princeton University

Princeton, NJ 08544

USA

E-mail address: bhargava@math.princeton.edu

DANIEL M. KANE

Department of Mathematics/

Department of Computer Science and Engineering

University of California, San Diego

LA Jolla, CA, 92093

USA

E-mail address: dakane@ucsd.edu

HENDRIK W. LENSTRA JR.

Mathematisch InstituUt

UNIVERSITEIT LEIDEN

Postbus 9512, 2300 RA Leiden

The Netherlands

E-mail address: hwl@math.leidenuniv.nl 


\section{BJORn PoOnen}

Department of Mathematics

Massachusetts Institute of Technology

CAmbridge, MA 02139-4307

USA

E-mail address: poonen@math.mit.edu

ERIC RAINS

Department of Mathematics

California Institute of Technology

Pasadena, CA 91125

USA

E-mail address: rains@caltech.edu

Received OCtober 15, 2014 